Supporting information for

\title{
Mesoscopic Simulation for the Effect of Cross-linking Reaction on the Drug Diffusion Properties in Microneedles
}

Yun Hao Feng, Xiao Peng Zhang, Liu Fu Hu, Bo Zhi Chen, Xin Dong Guo*

Beijing Laboratory of Biomedical Materials, College of Materials Science and Engineering, Beijing University of Chemical Technology, Beijing, 100029, P.R. China.

Corresponding author:

Xin Dong Guo, Beijing Laboratory of Biomedical Materials, College of Materials

Science and Engineering, Beijing University of Chemical Technology, Beijing, 100029, China. E-mail: xdguo@buct.edu.cn. 


\section{Calculation method of DPD repulsive parameters}

The most critical parameter is the repulsive parameter $a_{i j}$ between beads, and Groot and Warren have successfully established the relationship between this parameter and the Flory-Huggins parameter $\left(\chi_{i j}\right)$ in polymer physics ${ }^{1}$ :

$$
a_{i j}=a_{i i}+3.27 \chi_{i j}
$$

Generally, the repulsive parameters between two identical beads $a_{i i}$ are set to 78 (Reduced Unit). The repulsive parameters between different beads are calculated from the Flory-Huggins parameters, which can be obtained from the all-atom molecular dynamics simulation ${ }^{2}$ :

$$
\chi_{i j}=\frac{\Delta E^{m i x} V_{r}}{R T \varphi_{i} \varphi_{j} V}
$$

among them, $\varphi_{i}$ and $\varphi_{j}$ represent the volume fraction of $i$ beads and $j$ beads, respectively. $R$ and $T$ represent gas constant and temperature, which, in this study, is akin to 8.314 $\mathrm{J} \cdot \mathrm{mol}^{-1} \cdot \mathrm{K}^{-1}$ and $298 \mathrm{~K}$, respectively. $V$ is the total volume for the specific system and $V_{r}$ is the reference volume. $\Delta E^{m i x}$ is the mix energy and is calculated by ${ }^{3}$ :

$$
\Delta E^{m i x}=E_{i j}-\left(E_{i}+E_{j}\right)
$$

where $E_{i j}$ is the energy of binary blend systems, while $E_{i}$ or $E_{j}$ is the energy of pure component systems. For each kind of energy, several sorts of interaction are involved in ${ }^{4}$ :

$$
\begin{gathered}
E=E_{\text {bonded }}+E_{\text {nonbonded }}+E_{\text {cross }} \\
=\left(E_{\text {bond }}+E_{\text {angle }}+E_{\Phi}+E_{\chi}\right)+\left(E_{\text {vdw }}+E_{\text {coulombic }}\right)+E_{\text {cross }}
\end{gathered}
$$

where $E_{\text {bonded }}$ is the energy concerning about the bonded interaction such as the energy used to restrict the bond length $\left(E_{\text {bond }}\right)$, three-body angle $\left(E_{\text {angle }}\right)$, dihedral torsion angle 
$\left(E_{\Phi}\right)$ and the out of plane energy $\left(E_{\chi}\right) . E_{\text {nonbonded }}$ is the energy concerning about the nonbonded interaction such as van der waals interaction and the coulombic interaction, which is calculated by Ewald summation method (calculated with a cutoff of $18.5 \AA$, a spline width of $1 \AA$, a buffer width of $0.5 \AA$, and Ewald accuracy of $10^{-5} \mathrm{kcal} / \mathrm{mol}$ ). $E_{\text {cross }}$ refers to the energy in the cross term of potential equation ${ }^{5}$.

To introduce the method of parameter calculating, we take the calculation of the repulsive parameter between $\mathrm{C} 1$ bead and $\mathrm{C} 2$ bead as example. The all-atom structure of $\mathrm{C} 1$ bead and $\mathrm{C} 2$ bead have been shown in the Table R1. First, we have constructed the pure component systems and the binary blend system (For pure component system: number of molecule is 50 , density is $0.93 \mathrm{~g} / \mathrm{cm}^{3}$. For binary blend system: number of $\mathrm{C} 1$ and $\mathrm{C} 2$ is 20 , density is $0.74 \mathrm{~g} / \mathrm{cm}^{3}$ ).

The constructed systems were subjected to 10,000-step geometric optimization to relax the structures, and the lowest energy conformation was obtained by an annealing method between $300 \mathrm{~K}$ and $1000 \mathrm{~K}$. The conformation was subjected to NVE simulation to balance the energy of whole system, and was followed by 5 ns NVT simulation using a Velocity Scale thermostat with a temperature setting of $298 \mathrm{~K}$. The time step is $1 \mathrm{fs}$, and a frame of conformation and energy information is output every 5000 steps. Only the last ten frames are applied to statistic the energy information in order to make sure thermal equilibrium state. All the all-atom simulations are performed by MS 2019 software package with the forcefield of COMPASS II.

Through eq (3) \& (4), the average energy of the last ten frames of the simulation results is $1031.93 \mathrm{kcal} / \mathrm{mol}, 454.28 \mathrm{kcal} / \mathrm{mol}$ and $581.26 \mathrm{kcal} / \mathrm{mol}$ for the binary system, 
C1 pure system and C2 pure system, respectively. According to the eq (2), the FloryHuggins parameter equals -0.58 , so the DPD repulsive parameter is determined as 76.09 by eq (1).

\section{Note}

The Flory-Huggins parameters and DPD repulsive parameters between the various beads are listed in Table S1 and Table S2, respectively. More detailed calculation data of repulsive parameters can be found in "DPD repulsive parameters.xlsx" in “Corresponding Data.zip". 


\section{CG mapping method}

The monomer of HA is divided into three kinds of beads. Each cyclic skeleton structure is divided into three $\mathrm{H} 2$ beads, and the remaining side groups are defined as $\mathrm{H} 1$ bead (containing $\mathrm{N}$ atoms) and $\mathrm{H} 3$ bead (containing carboxyl group), respectively. For the ring structure, we used six $\mathrm{H} 2$ beads to maintain two ring structures (each ring contains three $\mathrm{H} 2$ beads) in the coarse-grained model, which may better reflect the natural structure of the all-atom structure than directly define it as one single bead ${ }^{6}$. Similar approaches have also been reported in a lot of previous DPD simulation studies ${ }^{7-8}$.

For SRB molecule, it is divided into three kinds of beads, S1 stands for the groups containing $\mathrm{N}$ atoms, and the S3 bead incarnates the sulfonyl groups. The structure similar to the benzene ring structure is symbolized as bead S2. To make sure the one bead would not containing too many atoms, we have divided this part as four beads for four rings ${ }^{9}$. Since there is rigid structure of SRB, we have fixed bond lengths (Figure S1A), three-body angles (Figure S1B). we have also constrained the bond angles S3$\mathrm{S} 2$ - S3 to highlight the geometric characteristics of 1,3 substitution (Figure S1C). Fixing these parameters can be achieved through the FIX function in the Material studio scripting code. For example :

\section{\{}

\# If you want to fix the bond length

my \$length $=$ \$doc-> UnitCell- $>$ Distance- $>$ “ ”; \# Input the length you want to fix. 
Fix("\$length")

\#If you want to fix the three-body angle

my $\$$ angle $=\$$ doc- $>$ UnitCell- $>$ Angle- $>$ “ ”; \# Input the length you want to fix.

Fix("\$angle")

\}

For BDDE molecule, we classify the hydroxyl group structure after ring-opening reaction as $\mathrm{C} 1$ beads and the hydrophobic carbon chain containing four carbon atoms as $\mathrm{C} 2$ beads. The $\mathrm{C} 1$ bead represent the group that can chemically react with the hydroxyl group of HA through ring-opening (the mechanism of crosslink reaction is shown in Movie S1) ${ }^{10}$. In order to abide by the approximate principle of the bead volume and mass, four water molecules are divided into one bead W. For this model, the average mass of each bead is $70.13 \mathrm{~g}$, and the average volume equals to $0.113 \mathrm{~nm}^{3}$ (Table S3).

The information about the bead classification and the corresponding all-atom structure can be seen in Table S4. 


\section{Calculation method of diffusion coefficients}

In this article, simple diffusion model is applied to study the drug diffusion. In this theory, the diffusion coefficient is calculated from the mean square displacement (MSD), and the relationship between the MSD and the diffusion coefficient is ${ }^{11}$ :

$$
2 n D=\frac{d M S D(t)}{d t}
$$

where $D$ is the diffusion coefficient and the $n$ denotes the dimension of the space. Since our simulation systems are three-dimension boxes, so the $n$ is akin to 3 . In our simulation, the script of the HA cross-linking reaction is completed randomly by searching bead distance in the simulation box and did not orient a specific direction. In the experiment, we also did not impose directional restrictions on the cross-linking reaction, so the occurrence of the cross-linking reaction should be isotropic. Therefore, we adopted the radial displacement $r$ of each bead relative to its initial position to calculate the $\mathrm{MSD}^{12}$ :

$$
M S D=\frac{1}{N} \sum_{i=1}^{N}<\left[r_{i}(t)-r_{0}(t)\right]^{2}>
$$

Hence, the MSD information should be obtained from the molecular dynamics simulation. There are three main approaches for obtaining the diffusion coefficient by MSD:

The first method is to numerically solve the first derivative of MSD concerning time. If the first derivative converges to a specific value, the diffusion coefficient is calculated according to this value. However, due to the limitations of the numerical method, it may cause a more significant error, as shown in the following figure. 


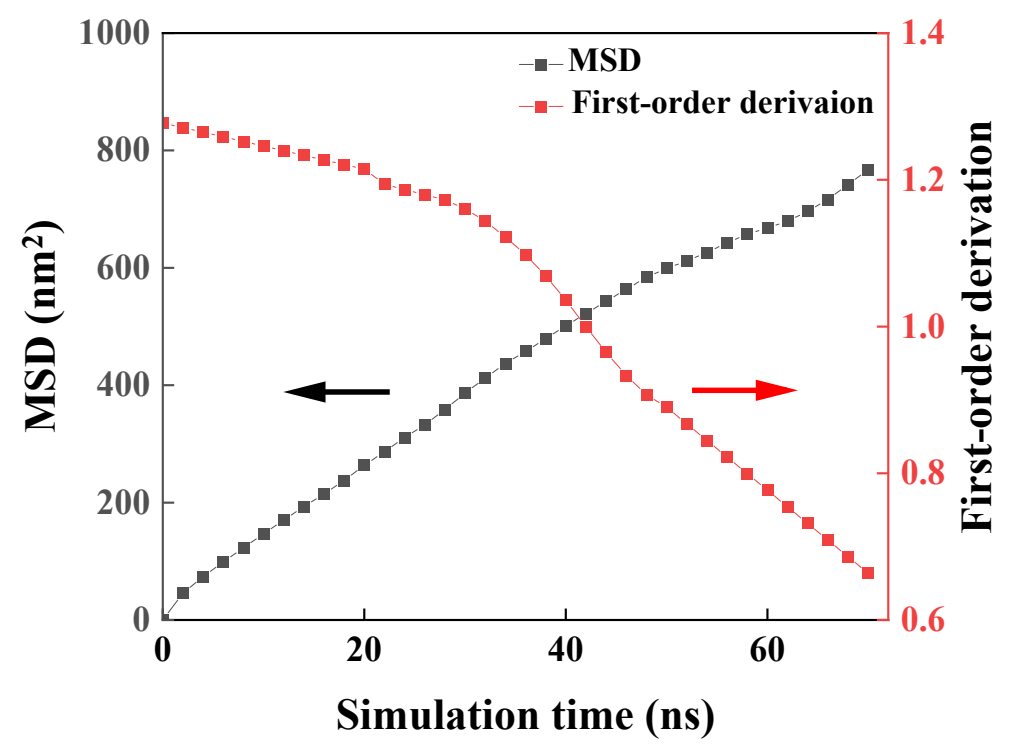

The second method is to perform linear fitting on MSD-time through the origin, and the diffusion coefficient could be attained from the slope of the linear fitting function, but it is required to find a reasonable fitting range. For one thing, the initial part of MSD data, which belongs to the diffusion relaxation process, cannot be used since the initial part unless the specific study purpose. For another, the last part of the MSD data cannot be used either, since the more significant the correlation time, the fewer data points, and the greater the MSD error. Therefore, as shown in the following figure, only the near-linear part of the data can be taken during fitting.

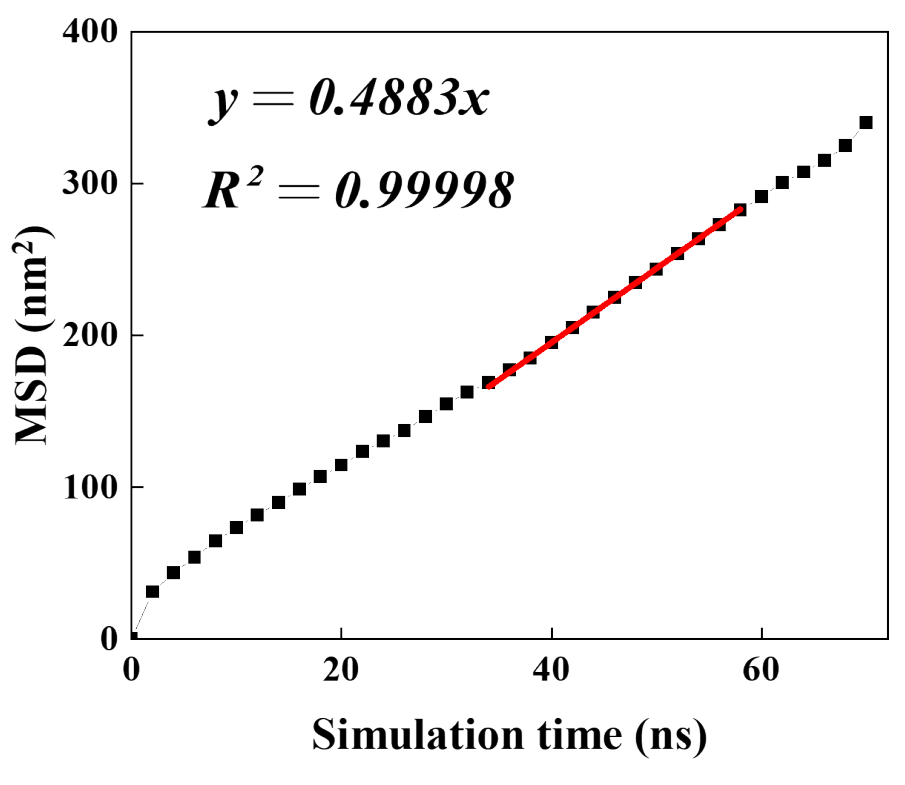


With the assistance of the following equation to analyze the logarithmic relationship between MSD and time:

$$
\ln (M S D)=\ln (t)+\ln (6 D)
$$

it is supposed to select the data range with a slope close to 1 (as shown in the figure), and calculate the diffusion coefficient by the intercept of the fitted straight line.

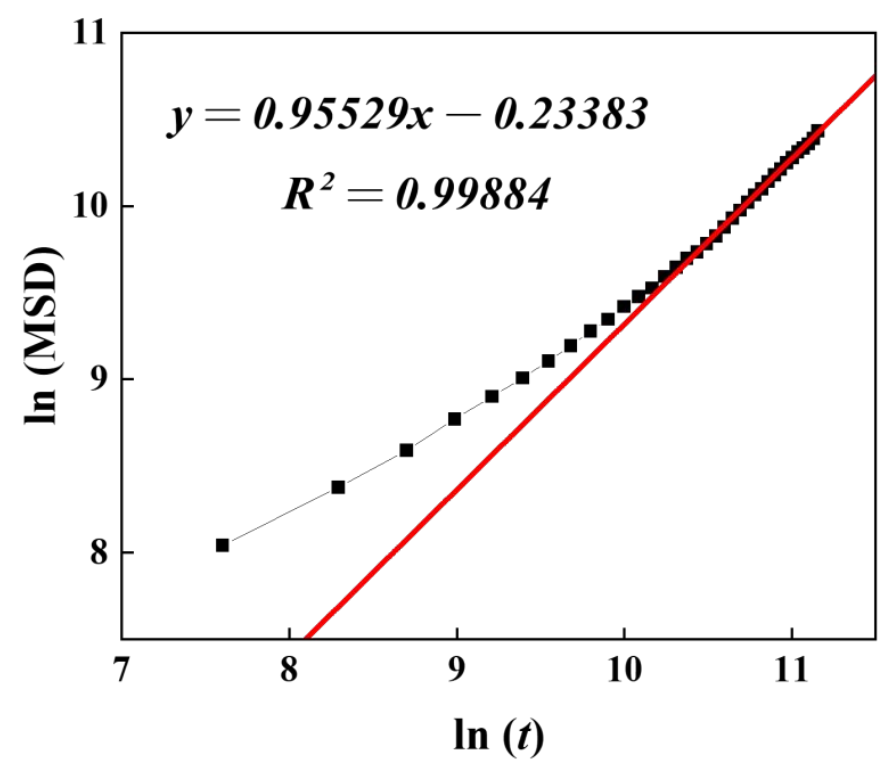




\section{Supplementary Figures}
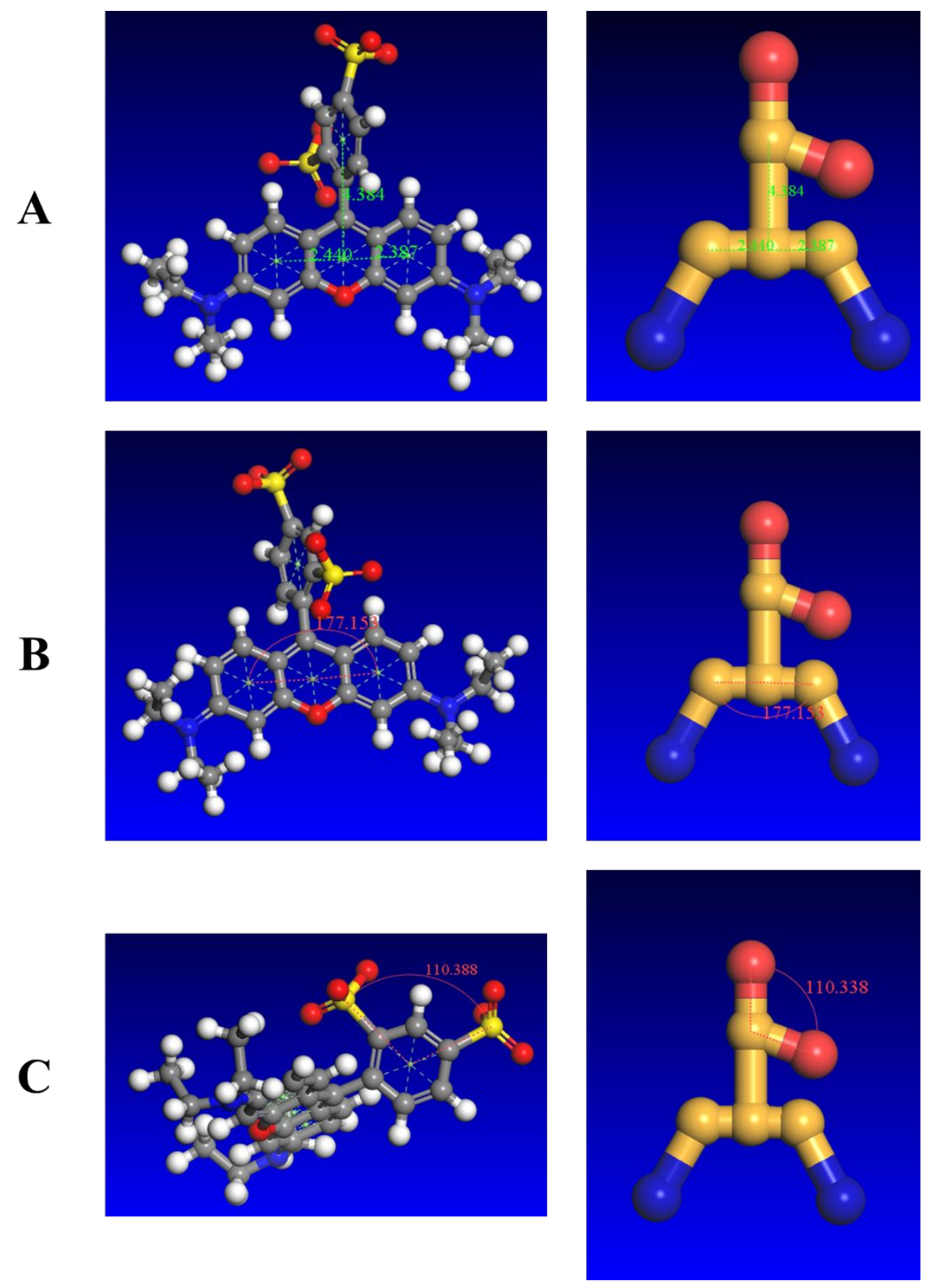

Figure S1. The geometric restrictions of the molecular structure related to SRB: A. Bond length. B. Bond angle (S2-S2-S2). C. Bond angle (S3-S2-S3). (The images on the left are the all-atom structure, while those on the right is the corresponding coarse-grained model, and the unit of size is $\AA$ ). 

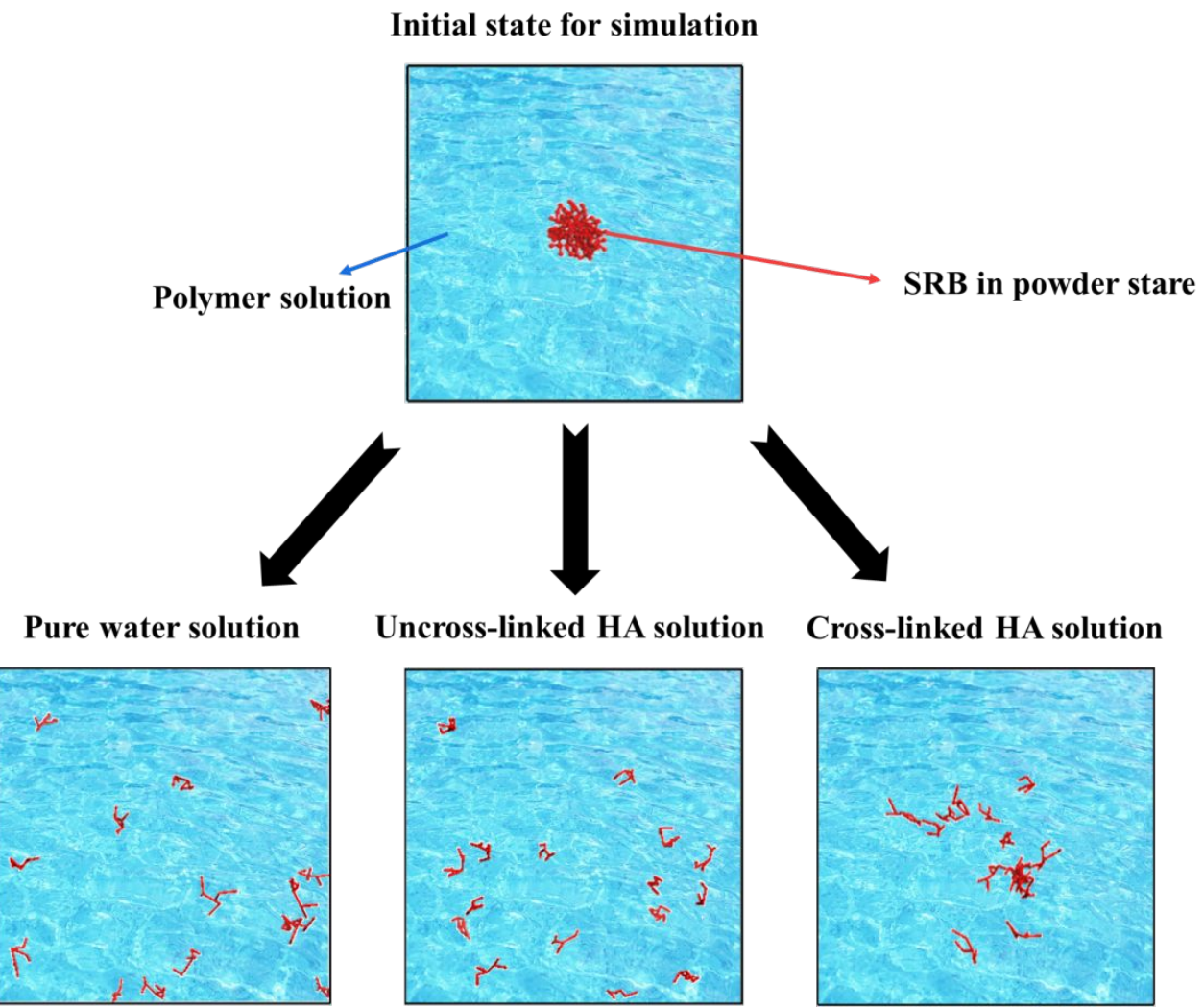

Cross-linked HA solution

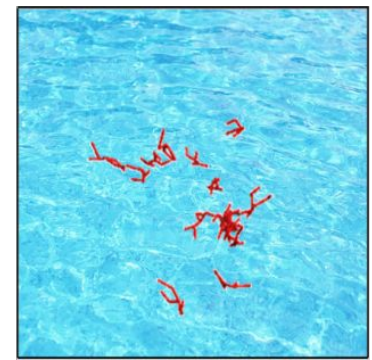

Figure S2. Schematic diagram of SRB diffusion in different solutions, HA and water beads are not shown for clarity. 


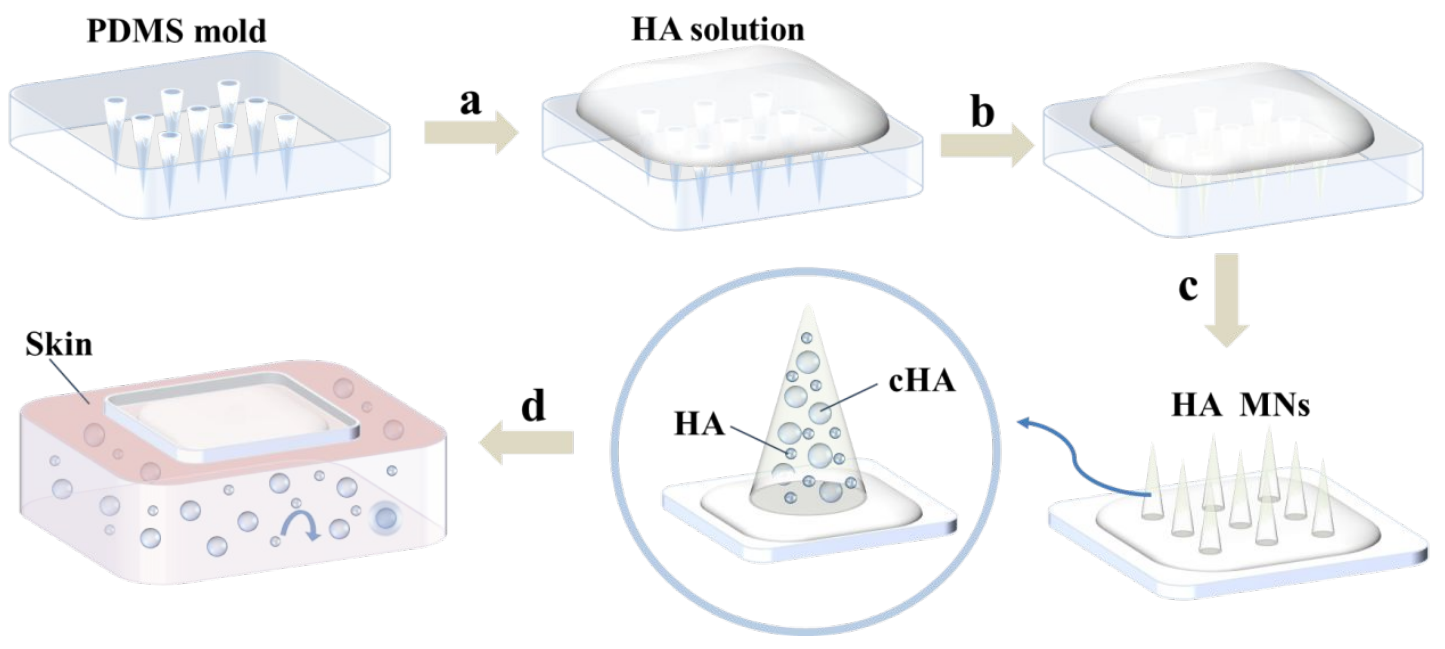

Figure S3. Schematic illustrations of fabrication process of HA-cHA composite MNs. 

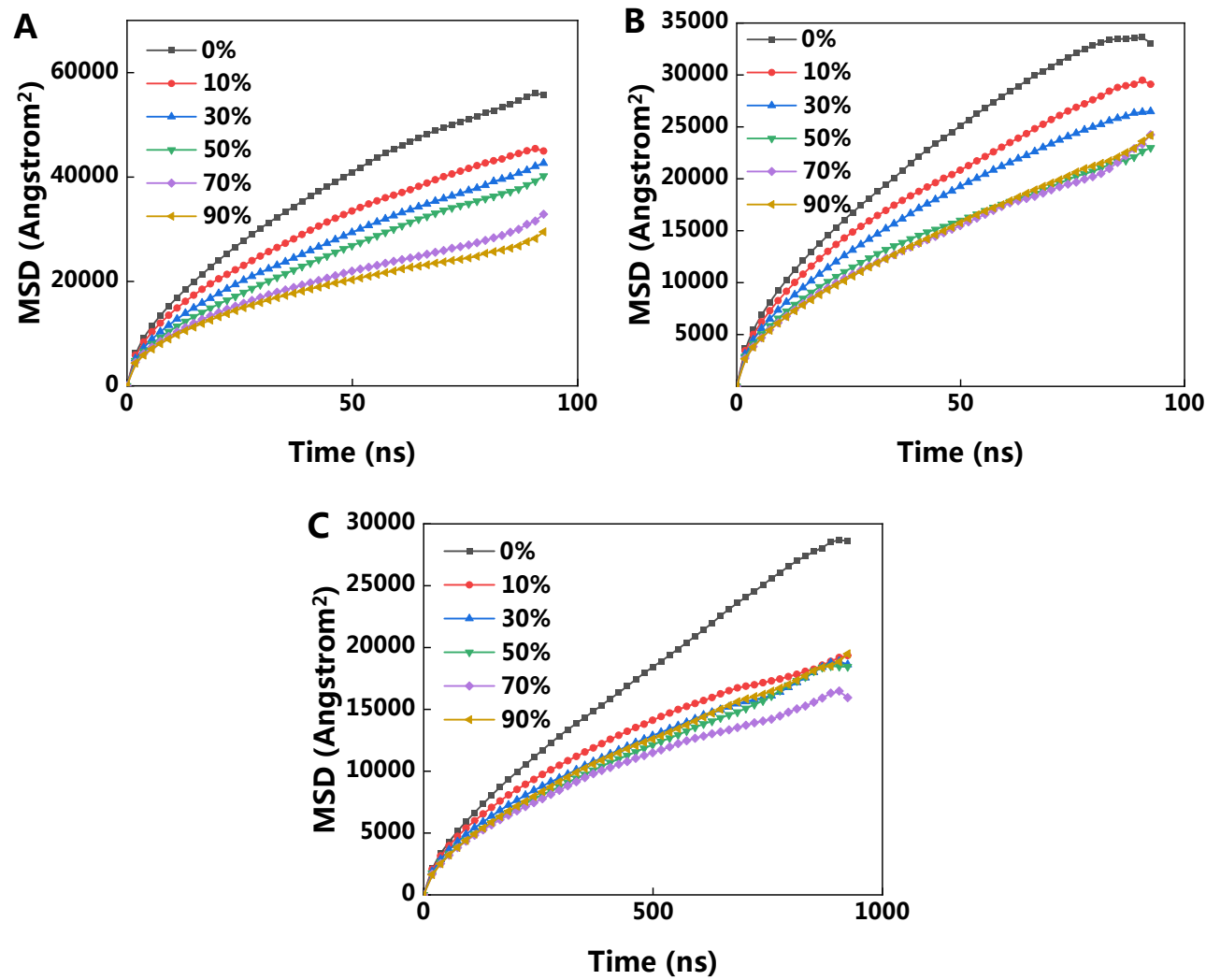

Figure S4. Mean square displacement of $\mathrm{H} 2$ beads in solutions with various concentrations. A. $10 \%(\mathrm{w} / \mathrm{w})$. B. $20 \%(\mathrm{w} / \mathrm{w})$. C. $30 \%(\mathrm{w} / \mathrm{w})$. 

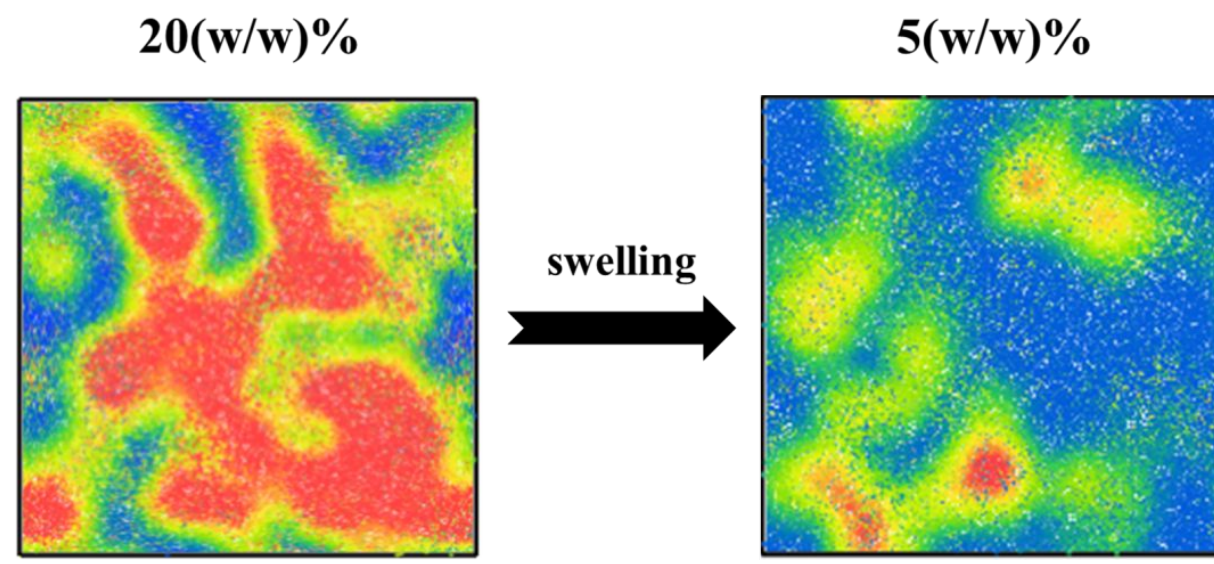

0.24505

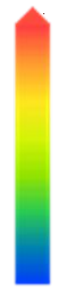

$\mathbf{0 . 0}$

Figure S5. Density field images of $\mathrm{H} 2$ beads in high concentration system (left) and low concentration system (right), and the cross-linking degree is set to $90 \%$. The left image and the Figure 6A4 in the manuscript are the same images. 


\section{Supplementary Tables}

Table S1. Flory-Huggins parameters between beads used in this simulation.

\begin{tabular}{|c|c|c|c|c|c|c|c|c|c|}
\hline & H1 & $\mathrm{H} 2$ & H3 & S1 & $\mathrm{S} 2$ & S3 & $\mathrm{C} 1$ & $\mathrm{C} 2$ & W \\
\hline H1 & 0.00 & & & & & & & & \\
\hline $\mathrm{H} 2$ & -2.05 & 0.00 & & & & & & & \\
\hline H3 & -20.52 & -21.12 & 0.00 & & & & & & \\
\hline $\mathrm{S} 1$ & -0.85 & -1.25 & -20.66 & 0.00 & & & & & \\
\hline $\mathrm{S} 2$ & -0.05 & -0.79 & -8.47 & -0.65 & 0.00 & & & & \\
\hline $\mathrm{S} 3$ & -20.39 & 41.23 & -17.93 & -18.13 & -14.89 & 0.00 & & & \\
\hline $\mathrm{C} 1$ & -15.83 & -1.53 & -19.16 & -20.83 & 0.32 & -15.50 & 0.00 & & \\
\hline $\mathrm{C} 2$ & 1.18 & -1.05 & -15.83 & -18.60 & 0.06 & -10.29 & -0.58 & 0.00 & \\
\hline W & 3.12 & -8.90 & -18.27 & 8.14 & -2.15 & -18.64 & 1.76 & 1.88 & 0.00 \\
\hline
\end{tabular}


Table S2. DPD repulsive parameters between beads used in this simulation (DPD Units)

\begin{tabular}{|c|c|c|c|c|c|c|c|c|c|}
\hline & H1 & $\mathrm{H} 2$ & H3 & S1 & $\mathrm{S} 2$ & S3 & $\mathrm{C} 1$ & $\mathrm{C} 2$ & W \\
\hline H1 & 78.00 & & & & & & & & \\
\hline $\mathrm{H} 2$ & 71.30 & 78.00 & & & & & & & \\
\hline H3 & 10.91 & 8.95 & 78.00 & & & & & & \\
\hline $\mathrm{S} 1$ & 75.20 & 73.92 & 10.45 & 78.00 & & & & & \\
\hline $\mathrm{S} 2$ & 77.84 & 75.42 & 50.31 & 75.88 & 78.00 & & & & \\
\hline $\mathrm{S} 3$ & 11.31 & 212.81 & 19.37 & 18.71 & 29.30 & 78.00 & & & \\
\hline $\mathrm{C} 1$ & 26.23 & 72.98 & 15.36 & 9.87 & 79.06 & 27.33 & 78.00 & & \\
\hline $\mathrm{C} 2$ & 81.85 & 74.58 & 26.22 & 17.18 & 78.19 & 44.34 & 76.09 & 78.00 & \\
\hline $\mathrm{W}$ & 88.19 & 48.91 & 18.24 & 104.62 & 70.98 & 17.04 & 83.76 & 84.15 & 78.00 \\
\hline
\end{tabular}


Table S3. Basic information of each bead

\begin{tabular}{|c|c|c|c|}
\hline & $\begin{array}{l}\text { volume of } 60 \\
\text { beads }\left(\AA^{3}\right)\end{array}$ & $\begin{array}{l}\text { volume of per } \\
\text { bead }\left(\AA^{3}\right)\end{array}$ & mass of bead $(\mathrm{g})$ \\
\hline $\mathrm{H} 1$ & 5777.59 & 96.29 & 59.00 \\
\hline $\mathrm{H} 2$ & 3626.82 & 60.45 & 46.67 \\
\hline H3 & 5996.78 & 99.95 & 59.00 \\
\hline $\mathrm{S} 1$ & 7162.16 & 119.37 & 87.00 \\
\hline $\mathrm{S} 2$ & 5134.26 & 85.57 & 64.50 \\
\hline $\mathrm{S} 3$ & 7172.92 & 119.55 & 95.00 \\
\hline $\mathrm{C} 1$ & 8979.07 & 149.65 & 90.00 \\
\hline $\mathrm{C} 2$ & 9705.87 & 161.76 & 58.00 \\
\hline $\mathrm{W}$ & 7479.55 & 124.66 & 72.00 \\
\hline average volume $\left(\AA^{3}\right)$ & 113.03 & average mass (g) & 70.13 \\
\hline cut-off radius $(\AA)$ & 6.97 & & \\
\hline
\end{tabular}


Table S4. CG mapping in this study

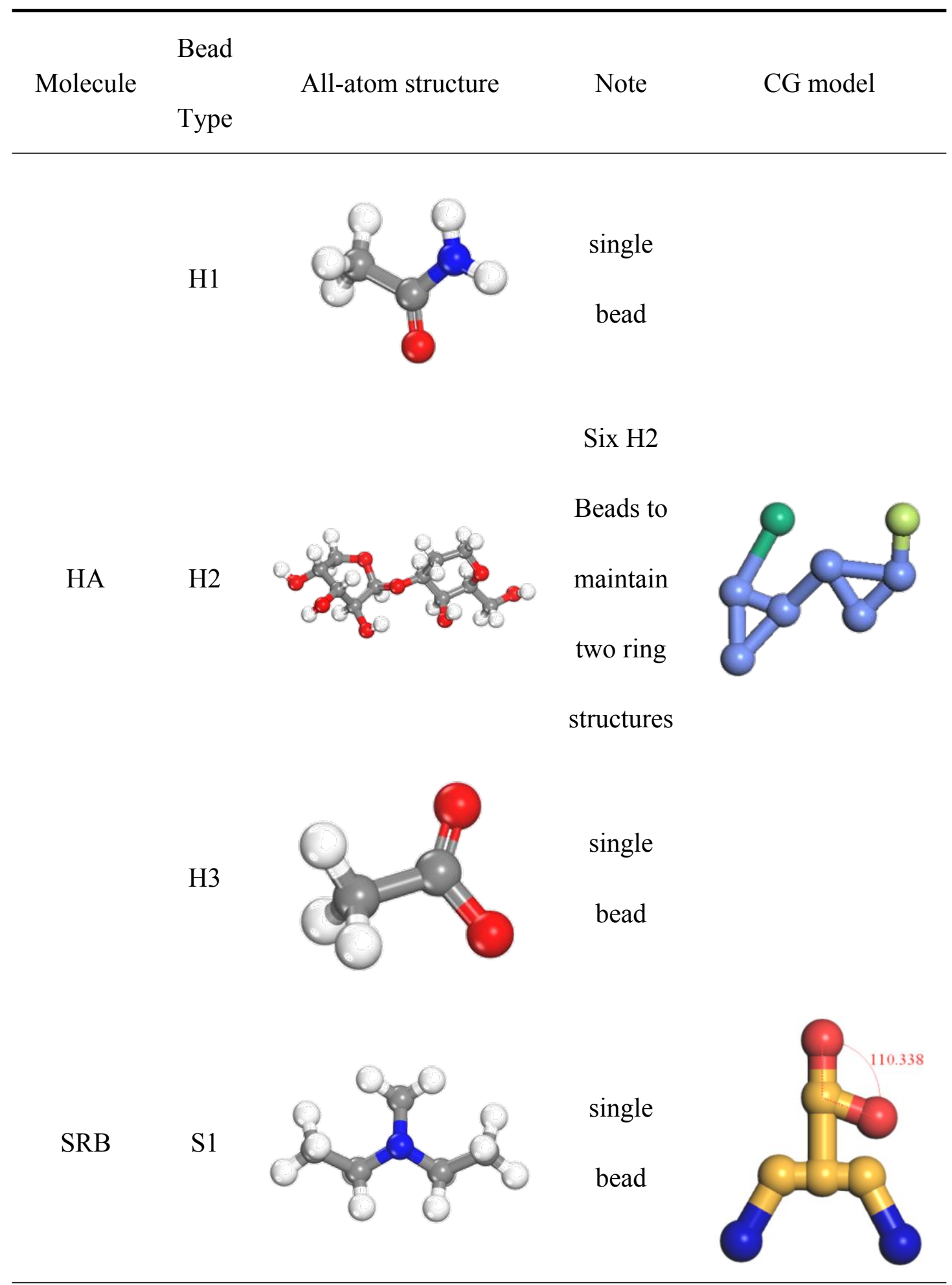


Table S4. CG mapping in this study (Continued)

S2

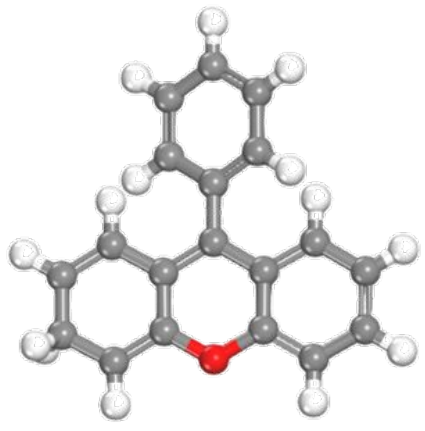

S3

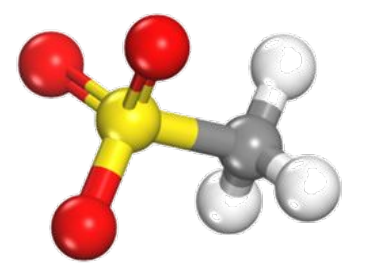

C1

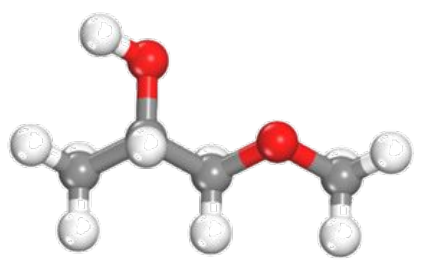

BEED

C2

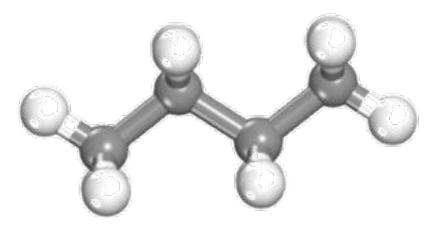

single bead

single bead

single bead

divided

into four

S2

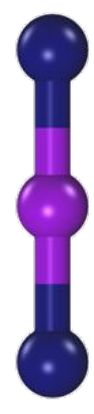

single bead solvent

W

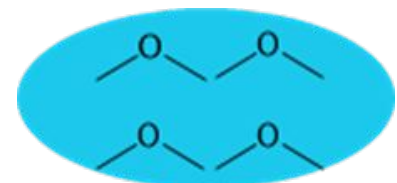


Table S5. Some data in the DPD Simulation systems

\begin{tabular}{|c|c|}
\hline Parameters & \\
\hline Temperature & $298 \mathrm{~K}$ \\
\hline Mass of bead & $70.13 \mathrm{~g}$ \\
\hline Cut-off Radius & $6.97 \AA$ \\
\hline Size of periodic cubic box & $209.1 \AA \times 209.1 \AA \times 209.1 \AA$ \\
\hline Total number of beads & 81000 \\
\hline Radius of SRB domain & $15 \AA$ \\
\hline Polymer concentration (mass ratio) & $10 \%-30 \%$ \\
\hline Total simulation time & $5 \times 10^{5}$ steps $(95 \mathrm{~ns})$ \\
\hline
\end{tabular}




\section{Topology of chemical system}

Note

All the chemical system documents are provided in "Topology of Chemical

System.zip" as ".xsd" format which are recommended to open in MS 2019 or other later versions.

Repeat unit of HA

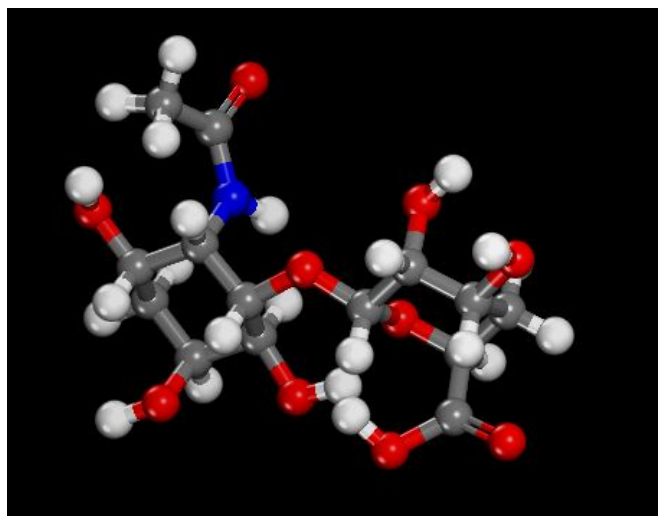

HA molecule of repeat degree of 10

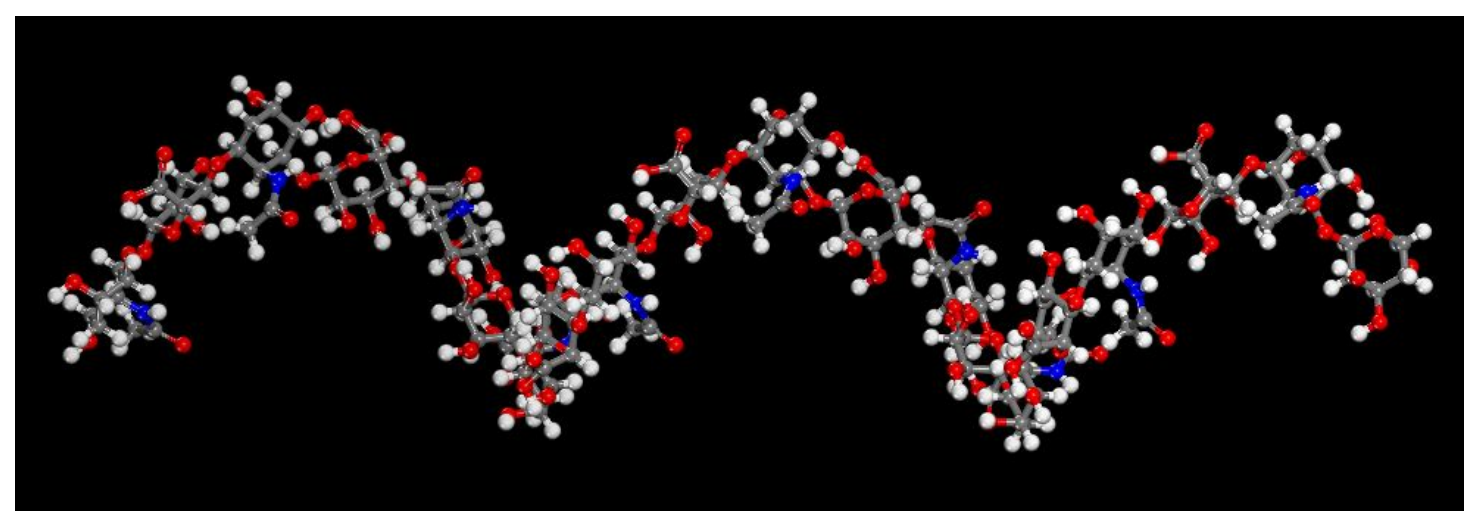

CG model of HA repeat unit 


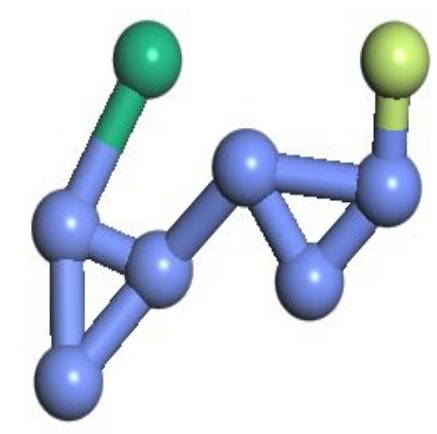

CG model of HA

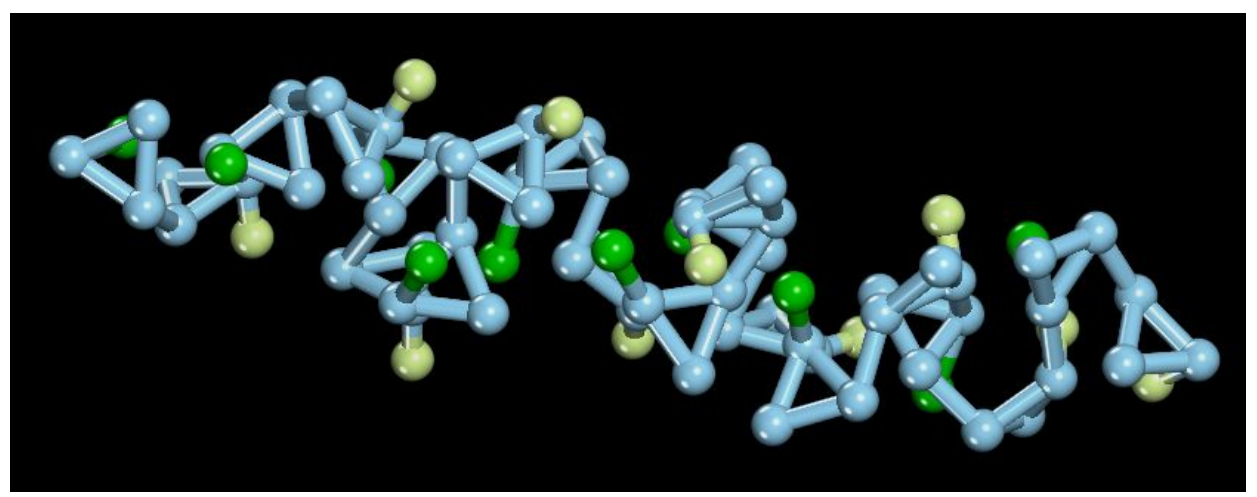

Crosslinking agent-BDDE

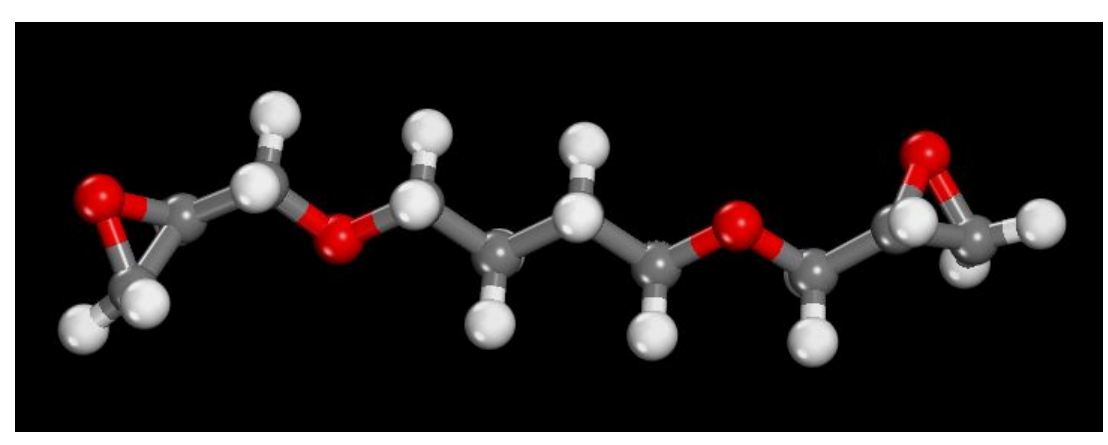

CG model of BDDE

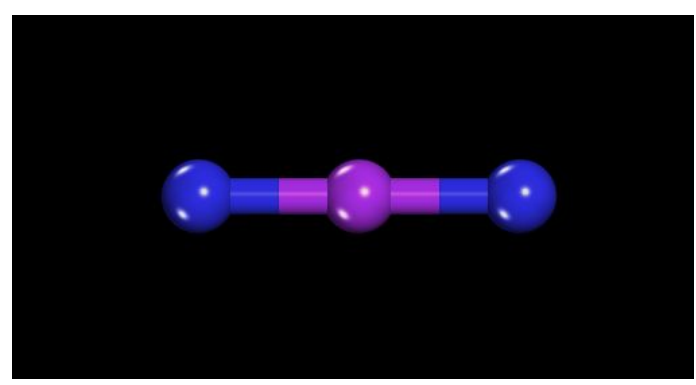

SRB 


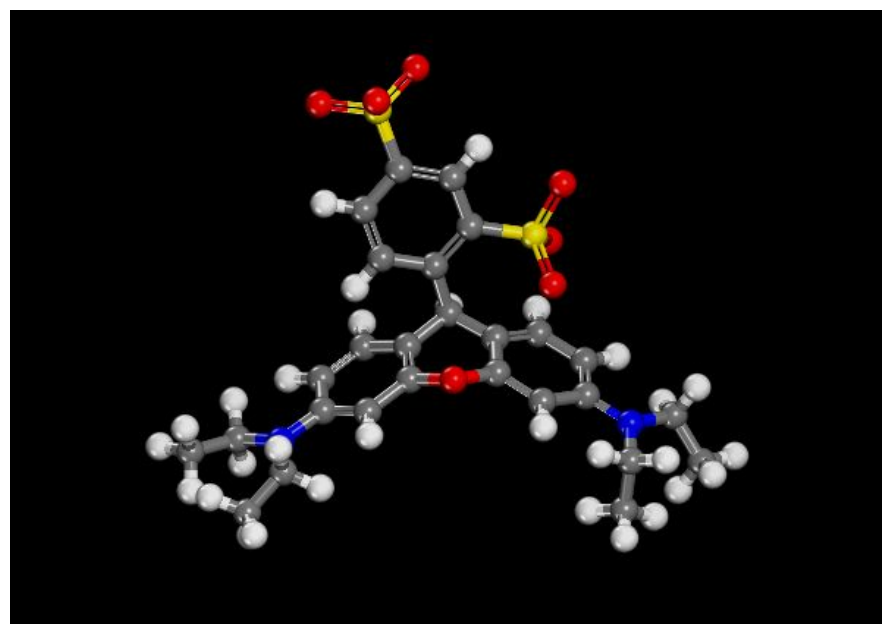

CG model of SRB

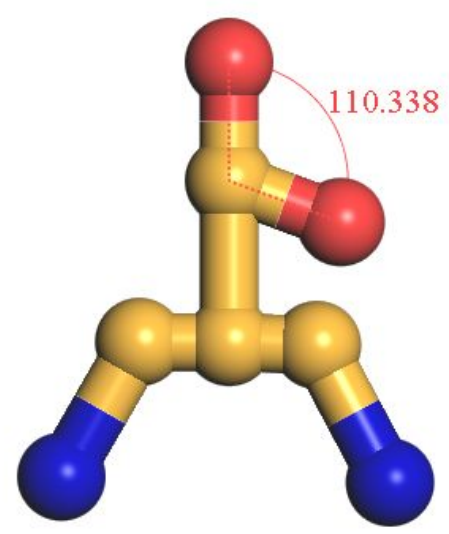

Chemical structure and CG model of water

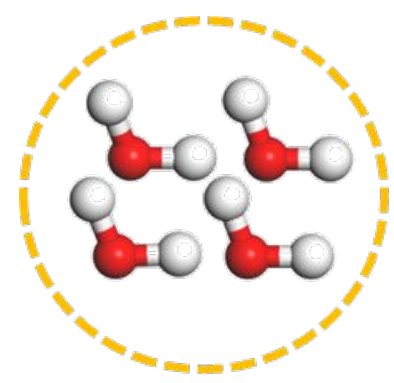

Water

\section{Cross-linking reaction: atom model}

The animation form is shown in the "Movie S1 Cross-linking reaction.avi" and "Cross-

linking reaction.xtd" in "Animation of Crosslink Reaction.zip". All the chemical 
system documents are provided in as ".xsd" format which are recommended to open in MS 2019 or other later versions.
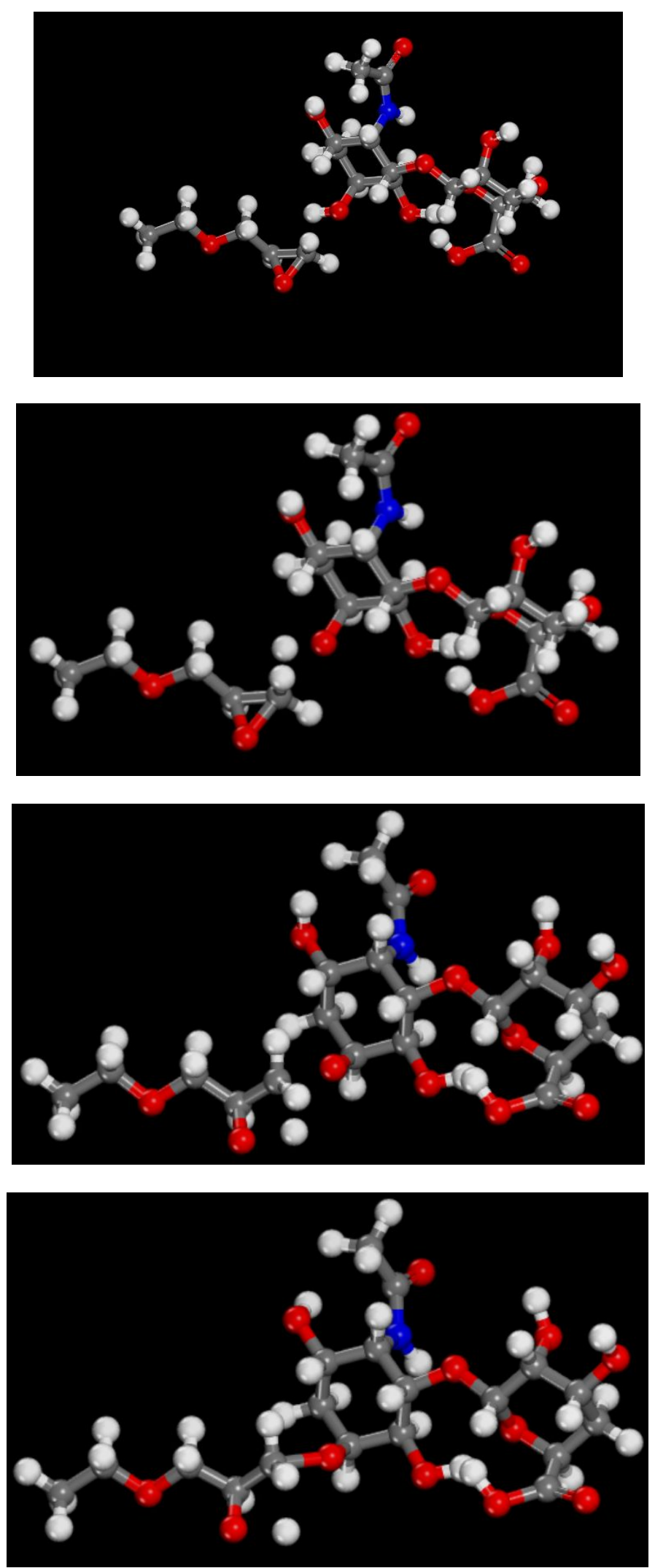


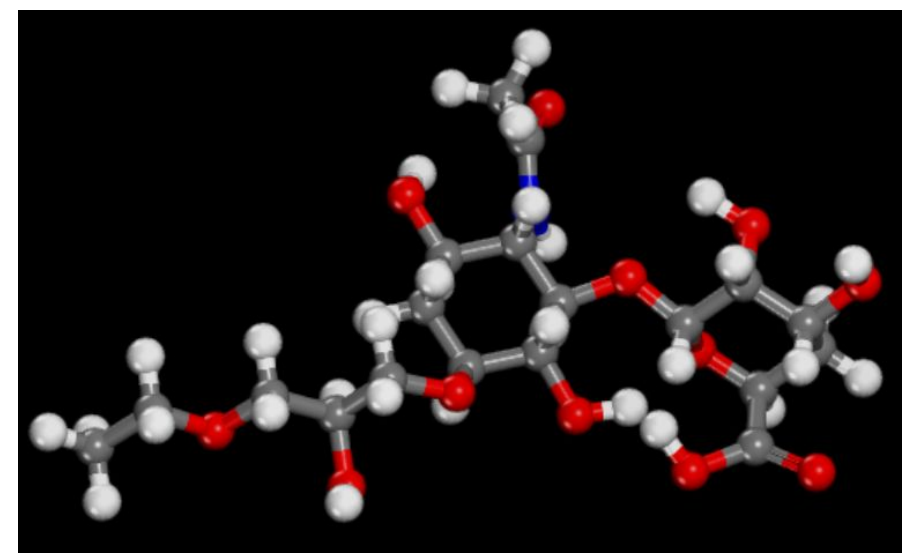

\section{Cross-linking HA solution}

Structure details are shown in "Cross-linking HA solution.xsd"

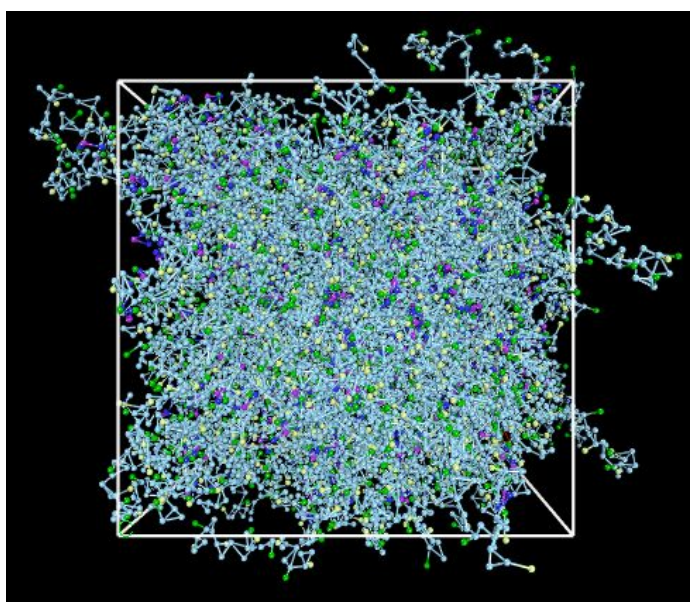

Solution

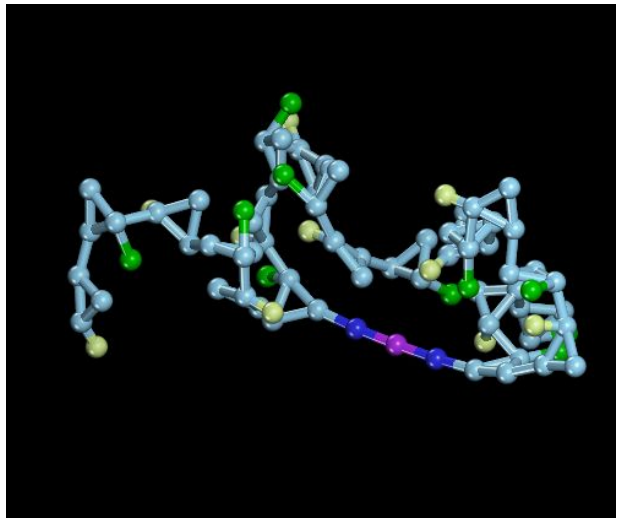

Intramolecular crosslinking 


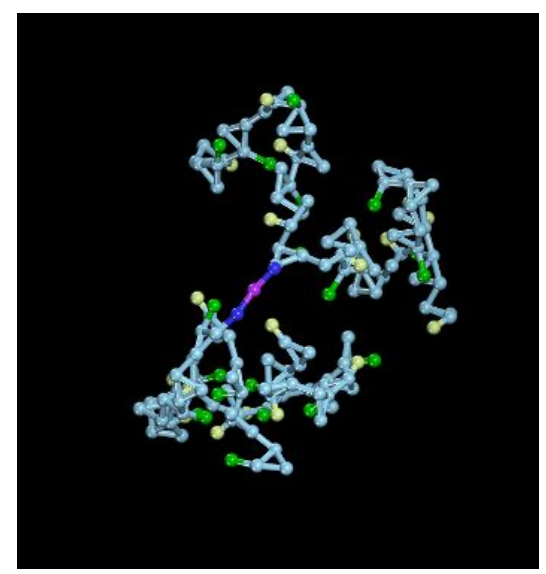

Intermolecular crosslinking 


\section{Corresponding code}

\section{Note}

All codes are written based on rules of Perl, and a large number of sub-functions in the code are provided by Material Studio software. It is recommended to run this code in MS 8.0 or other later versions. The ".pl" document are also provided in "Corresponding Code.zip".

\section{Code for basic simulation and analysis}

\#!perl

use strict;

use Getopt::Long;

use MaterialsScript qw(:all);

my $\$$ doc $=\$$ Documents $\{" "\} ; \quad$ \#input your subject document name

\#GeometryOptimization

my \$results $=$ Modules- $>$ Mesocite- $>$ GeometryOptimization- $>$ Run $(\$$ doc, Settings (

NonPeriodicvdWAtomCubicSplineCutOff $=>$ 6.97, \#cut-off radius

NonPeriodicvdWAtomCubicSplineWidth $=>0$,

CurrentForcefield => '/', \#input the DPD force field file name

MaxIterations $=>50000$,

OptimizationAlgorithm => 'Conjugate gradient',

MaxForce $=>0.001$,

MaxStress $=>0.001$,

MaxDisplacement $=>$ 1e-005, 
MaxEnergy $=>2 \mathrm{e}-005$,

UseMaxForce => 'Yes',

UseMaxStress $=>$ 'Yes',

UseMaxDisplacement => 'Yes',

ExternalPressure $=>0.0001)$;

\#DPD simulation

my \$results $=$ Modules- $>$ Mesocite- $>$ DPD- $>$ Run $(\$ d o c$, Settings (

NonPeriodicvdWAtomCubicSplineCutOff $=>$ 6.97, \#cut-off radius

NonPeriodicvdWAtomCubicSplineWidth $=>0$,

CurrentForcefield => '/', \#input the DPD force field file name

NumberOfSteps $=>500000$,

TimeStep $=>184.879, \quad$ \#Unit in fs

TrajectoryFrequency $=>1000$,

Dissipation $=>0.08486$,

DissipationRadius $=>6.97$, \#Unit in angstrom

Temperature $=>298$, \#Unit in $\mathrm{K}$

my $\$$ outTrajectory $=$ \$results- $>$ Trajectory;

\#The MSD, RDF and Rg analysis

\#MSD

my $\$$ results $=$ Modules- $>$ Mesocite- $>$ Analysis- $>$ MeanSquareDisplacement $(\$ d o c$, Settings (

MSDFrameOriginStep $=>1$, \#Choosing the ".xtd" document in simulation results

MSDMaxFrameLength $=>1000$, 
MSDSetA => ")); \#Choosing subject sets

my $\$$ outMSDChart $=$ results- $>$ MSDChart;

my \$outMSDChartAsStudyTable = \$results->MSDChartAsStudyTable;

\#RDF

my $\$$ results $=$ Modules $->$ Mesocite- $>$ Analysis $->$ RadialDistributionFunction $(\$ d o c$, Settings (

RDFBinWidth $=>0.05$, \#Choosing the ".xsd" document in simulation results

RDFCutoff $=>100$, \#Unit in angstrom

RDFSetA $=>$,

RDFSetB => ")); \#Choosing subject sets

my $\$$ outRDFChart $=\$$ results- $>$ RDFChart;

my $\$$ outRDFChartAsStudyTable $=$ \$results->RDFChartAsStudyTable;

\#Rg

my $\$$ results $=$ Modules->Mesocite->Analysis->RadiusOfGyration $(\$ d o c$, Settings (

\#Choosing the ".xsd" document in simulation results

RadiusOfGyrationSetA => ", \#Choosing subject sets

RadiusOfGyrationBinWidth $=>0.05$,

RadiusOfGyrationSmoothedPlot $=>$ 'Yes',

RadiusOfGyrationSmoothedWidth $=>4)$ );

my \$outRadiusOfGyrationChart $=$ \$results-> RadiusOfGyrationChart;

my \$outRadiusOfGyrationChartAsStudyTable $=$ \$results- $>$ RadiusOfGyrationChartAsStudyTable;

\section{Code for cross-linking reaction}

$\#$ !perl 
use strict;

use Getopt::Long;

use MaterialsScript qw(:all);

my $\$$ doc $=\$$ Documents $\{" "\} ;$ \#input your subject document name

my $\$$ Rnum $=0$;

my $\$$ Bondnum $=0$;

my \$Percent;

my $\$$ Degree $=0$;

my $\$ r=5$;

my $\$ \mathrm{R}=10$;

my@R1;

foreach my \$bead (@\{\{doc->UnitCell->Beads $\})$

\{

my $\$$ name $=$ \$bead->BeadTypeName;

if(\$name eq "R1")

\{

$\operatorname{push}(@ \mathrm{R} 1$, bead $)$;

++\$Rnum; \#Record the number of the possible reaction sites

\}\}

my@R2;

foreach my \$bead (@ $\{\$$ doc->UnitCell->Beads $\})$ 


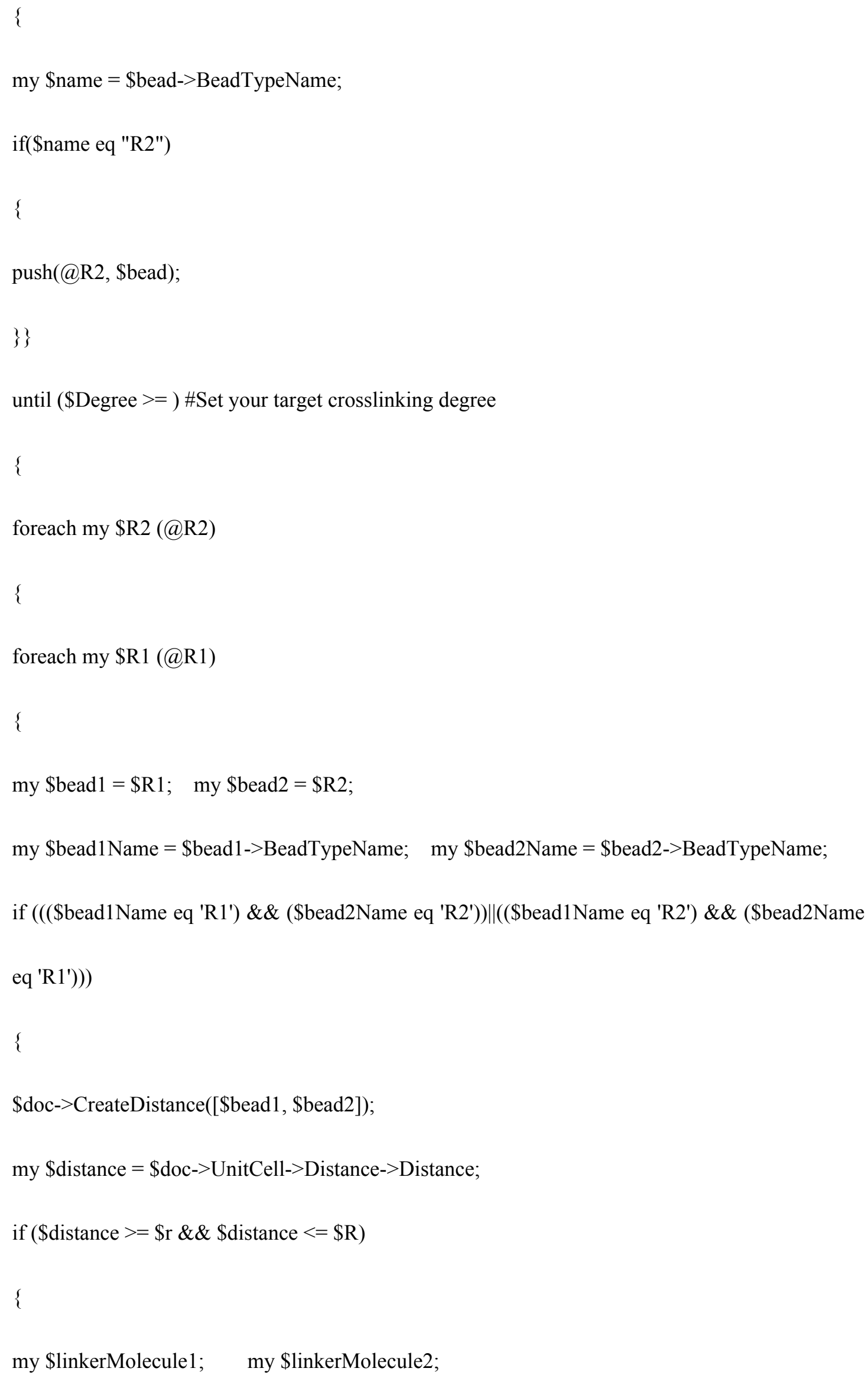




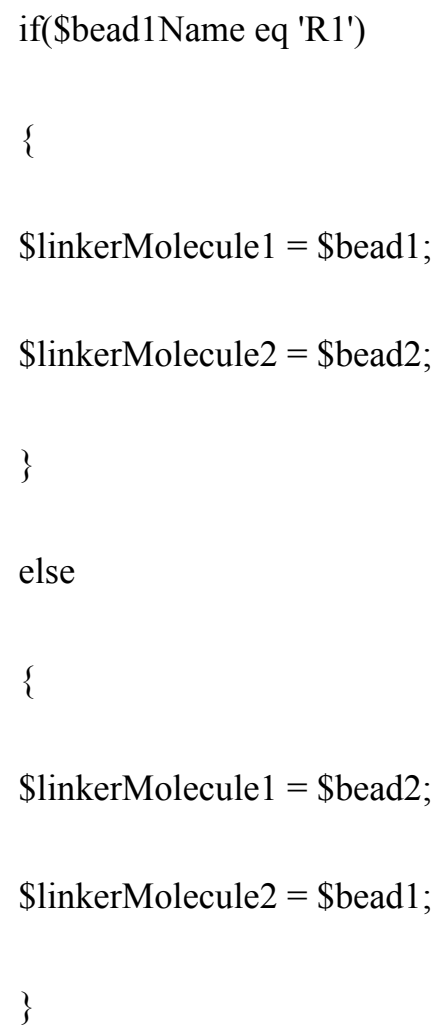


else

\$doc->CreateBeadConnector $(\$$ bead1, \$bead2);

\$linkerMolecule1->Ancestors->Molecule->Name = "\$linkerMoleculeName"."_Cross-linked";

++\$Bondnum;

\$R1->Ancestors->Bead->BeadTypeName = "H2";

\$linkerMolecule2->Ancestors->Molecule->Name = "\$linkerMoleculeName";

\$doc->UnitCell->Distances->Delete;

last;

\}\}

else

\{

\$doc->UnitCell->Distances->Delete;

\{\}$\}\}$

$\$$ Degree $=\$$ Bondnum $/ \$$ Rnum; $\#$ Calculate the corss-linking degree

$\$$ Percent $=\$$ Degree $* 100$;

printf "When the distance ranges from \%f angstrom to \% f angstrom $\backslash n$ the corss-linking degree of the system is $\% \mathrm{f} \% \backslash \mathrm{nn} "$, $\$ r, \$ R$, \$Percent;

$\$ r=\$ r+5$

$\$ \mathrm{R}=\$ \mathrm{R}+5$

\} 


\section{Available data}

\section{Note}

All the ".opju" (Origin file) and "xlsx" (Excel file) document are also provided in "Editable Origin Figure.zip" and "Corresponding Data.zip" respectively.

Set S1. Editable figure and original data of Figure 3A in the manuscript.

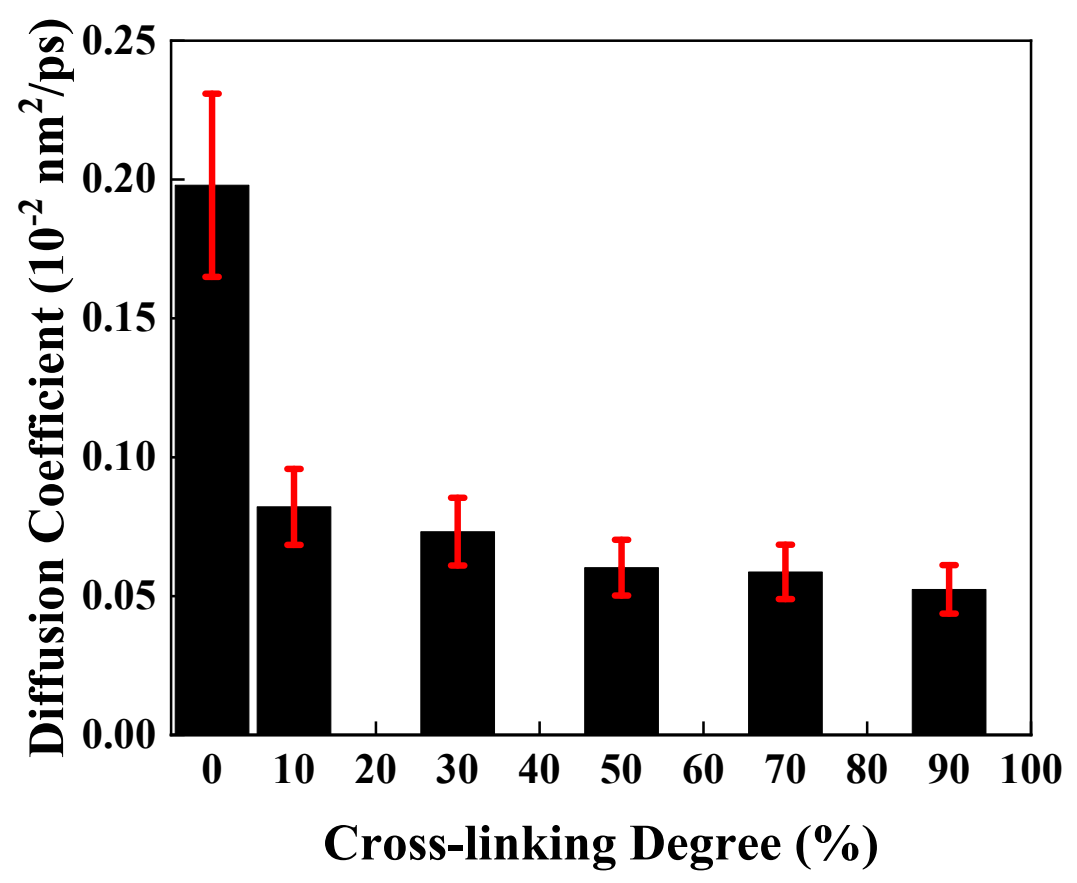


Set S2. Editable figure and original data of Figure 3B in the manuscript.

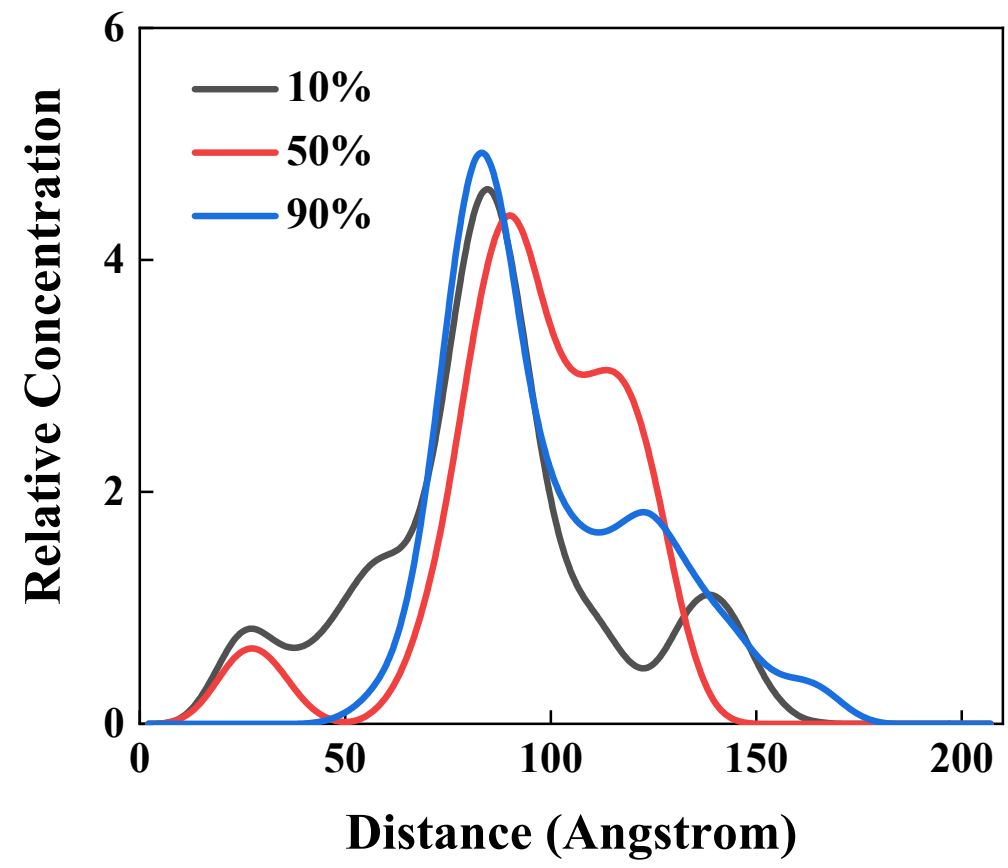


Set S3. Editable figure and original data of Figure 3C in the manuscript.

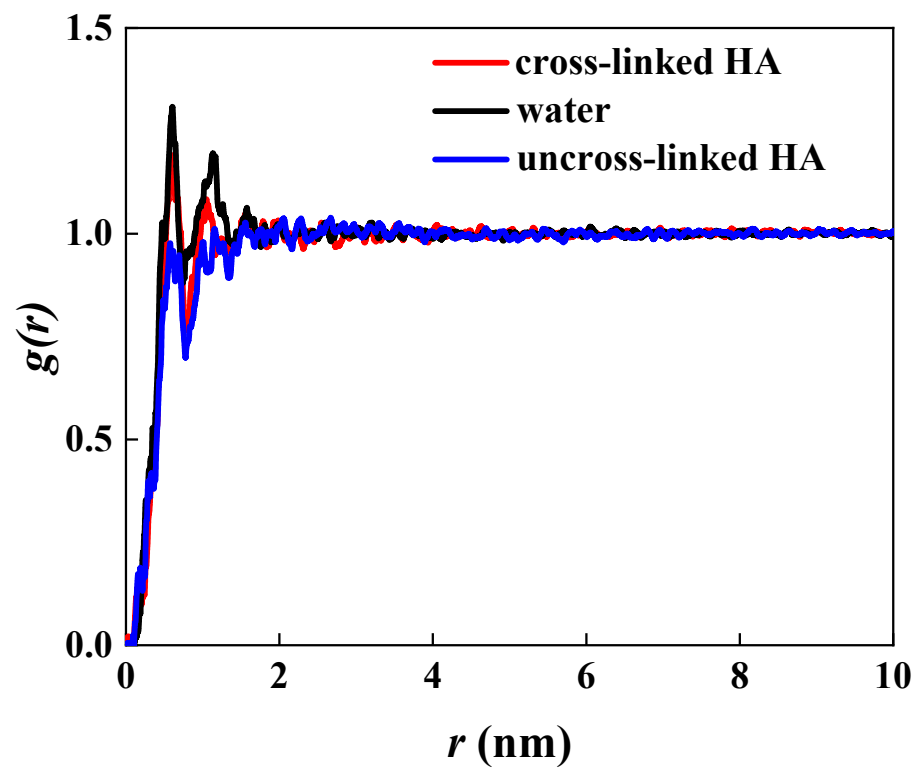


Set S4. Editable figure and original data of Figure 3D in the manuscript.

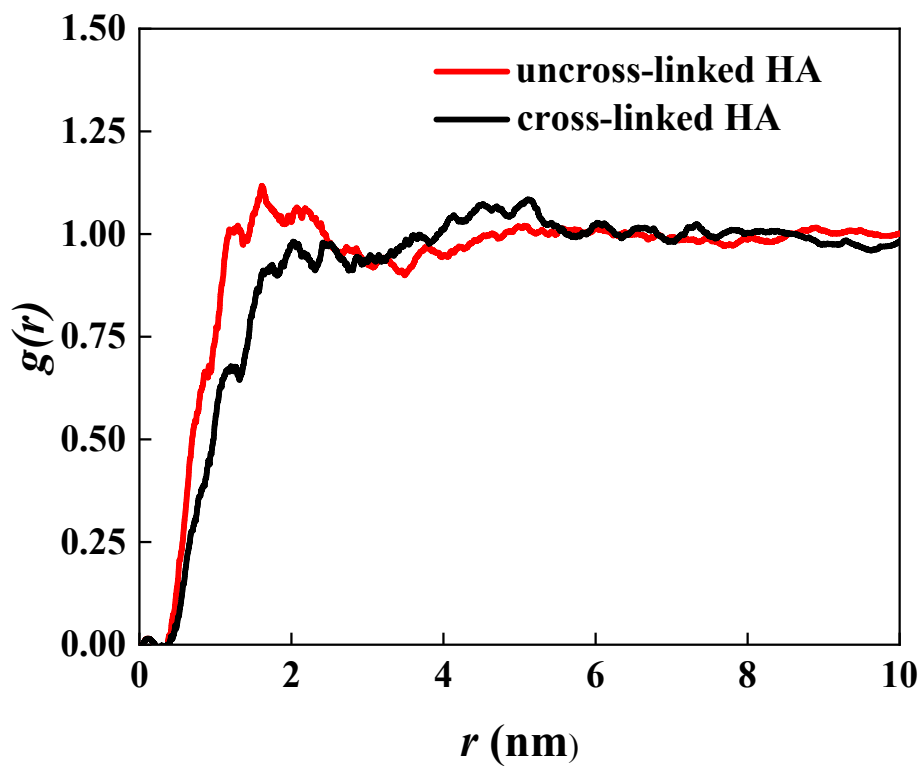


Set S5. Editable figure and original data of Figure 4A in the manuscript.

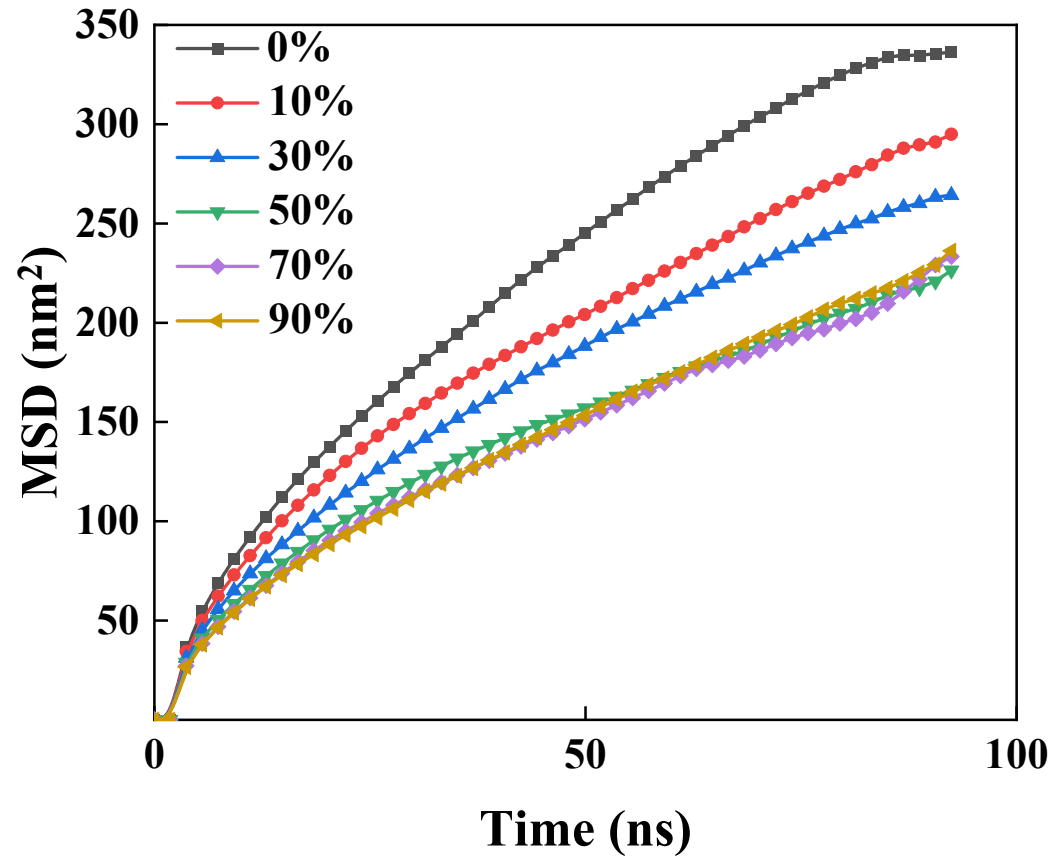


Set S6. Editable figure and original data of Figure 4B in the manuscript.

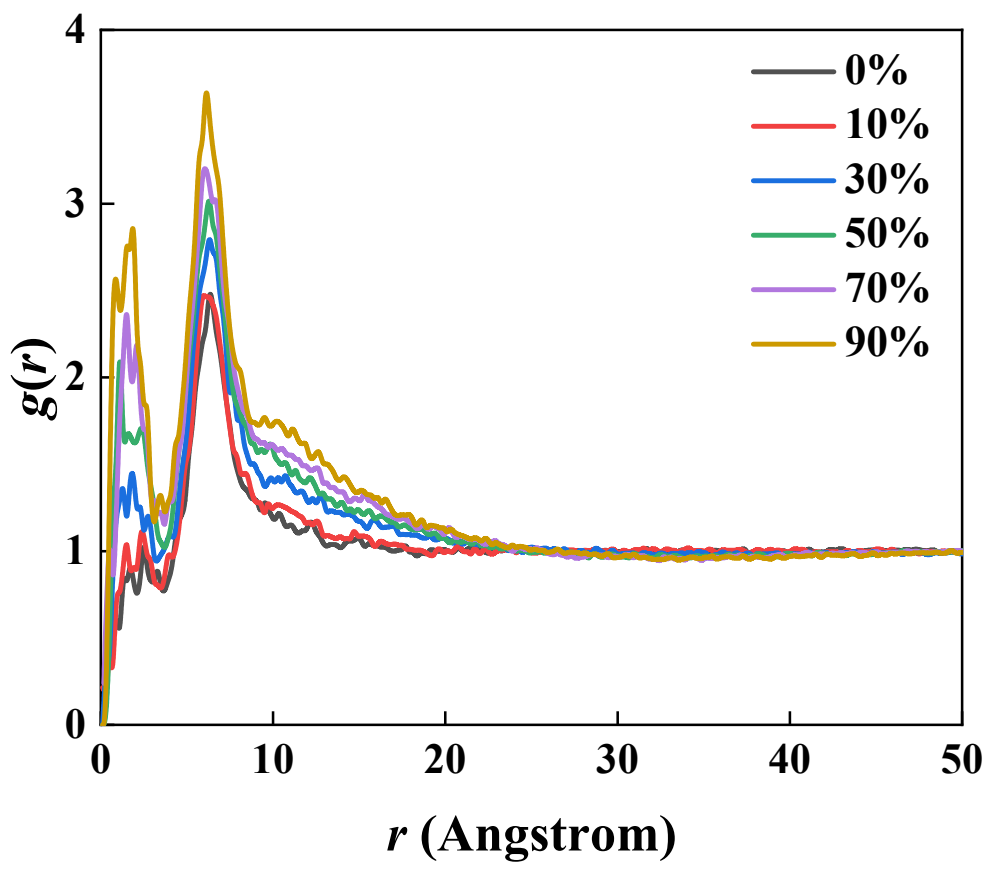


Set S7. Editable figure and original data of Figure 4C in the manuscript.
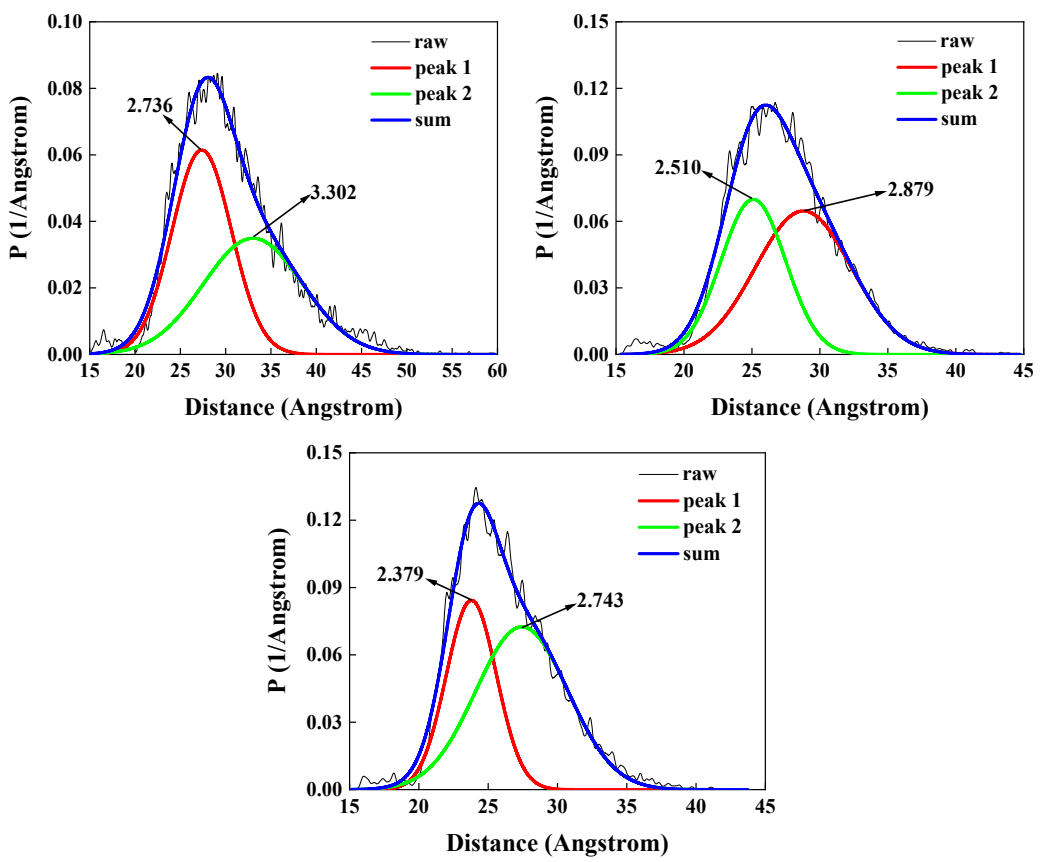
Set S8. Editable figure and original data of Figure 6B in the manuscript.

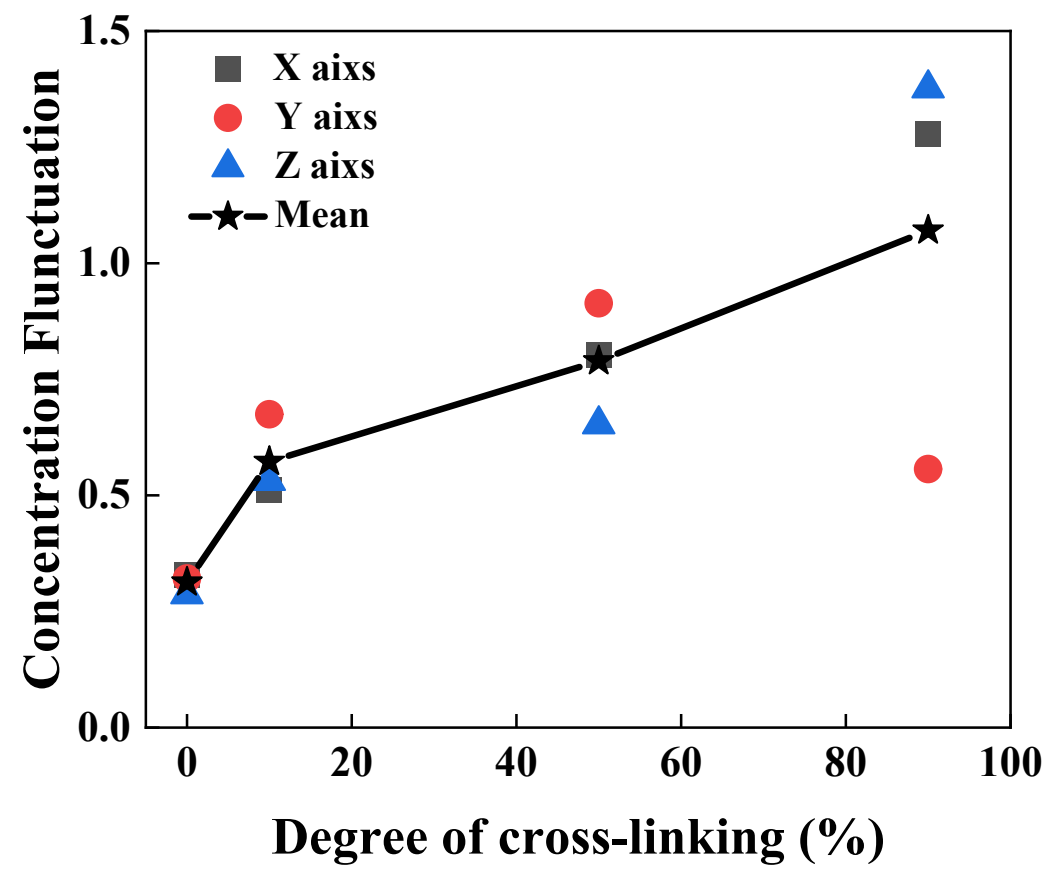


Set S9. Editable figure and original data of Figure 6C in the manuscript.
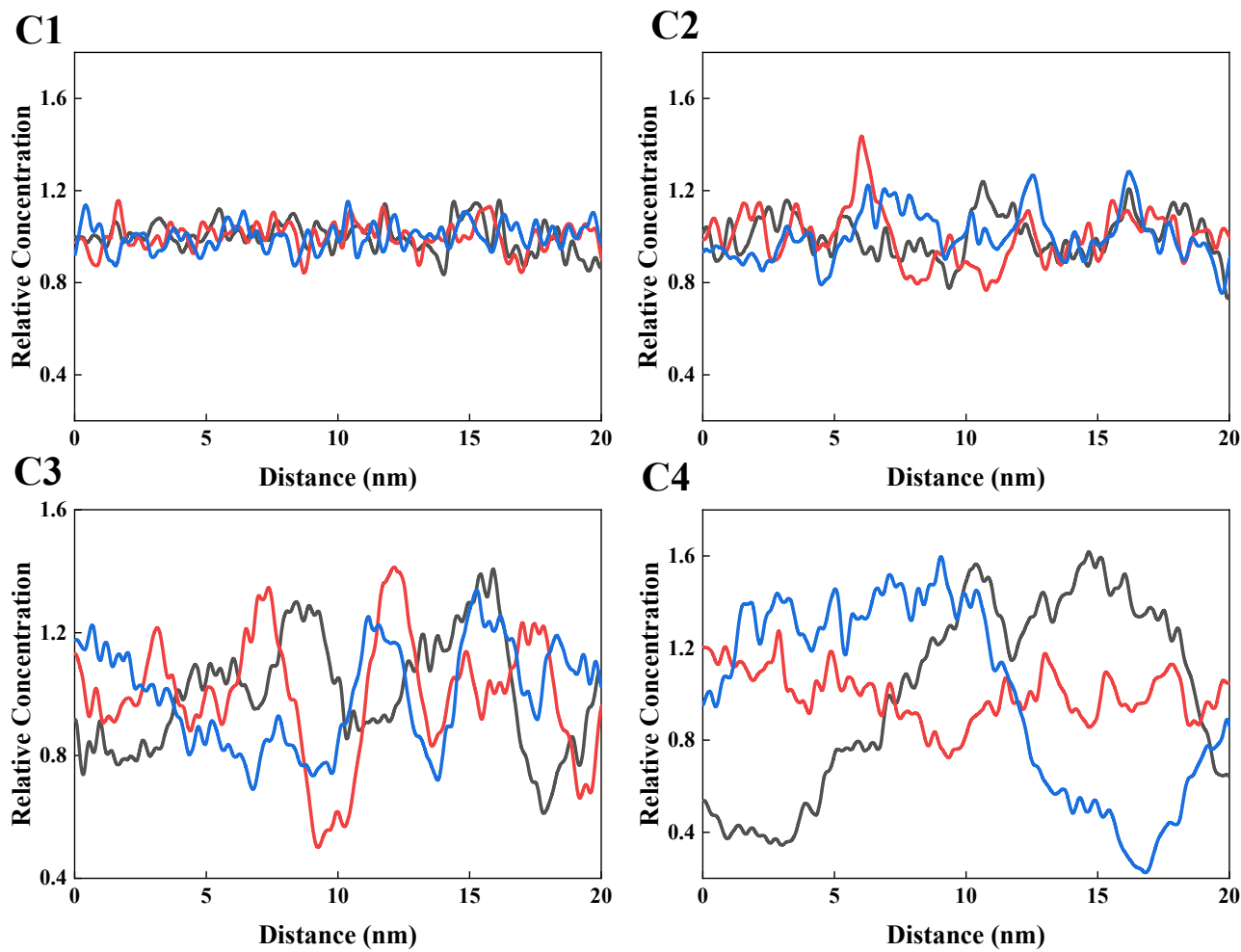
Set S10. Editable figure and original data of Figure 7A in the manuscript.

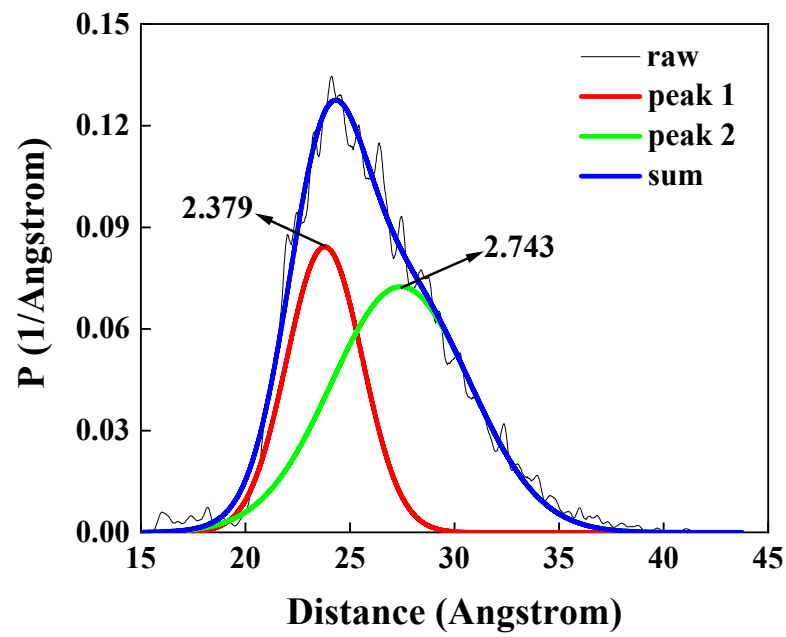


Set S11. Editable figure and original data of Figure 7B in the manuscript.

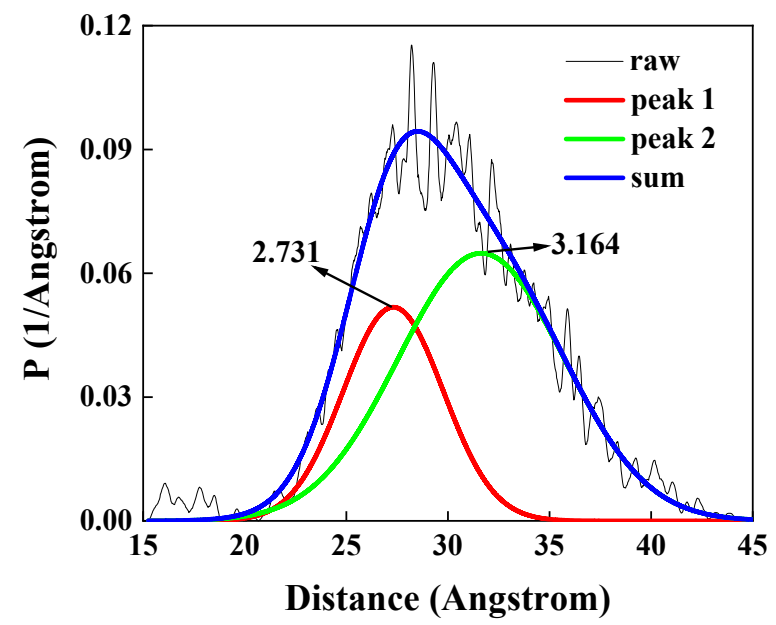


Set S12. Editable figure and original data of Figure 7C in the manuscript.

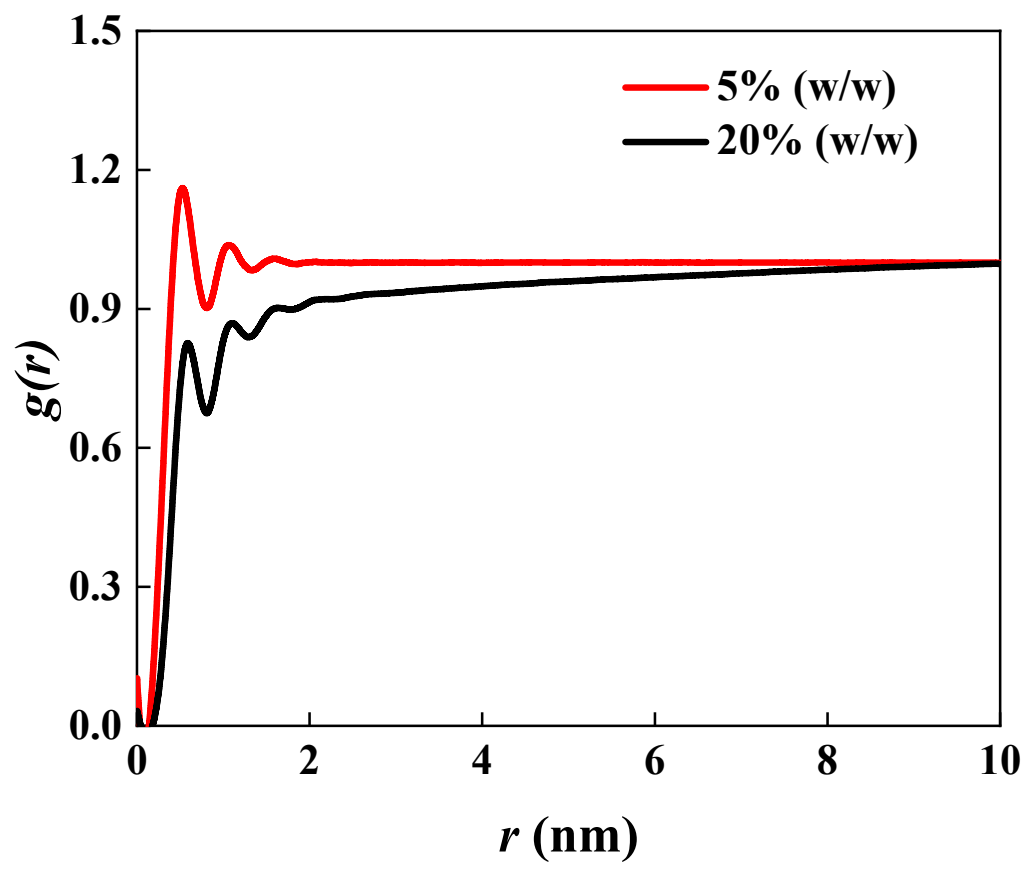


Set S13. Editable figure and original data of Figure 7D in the manuscript.

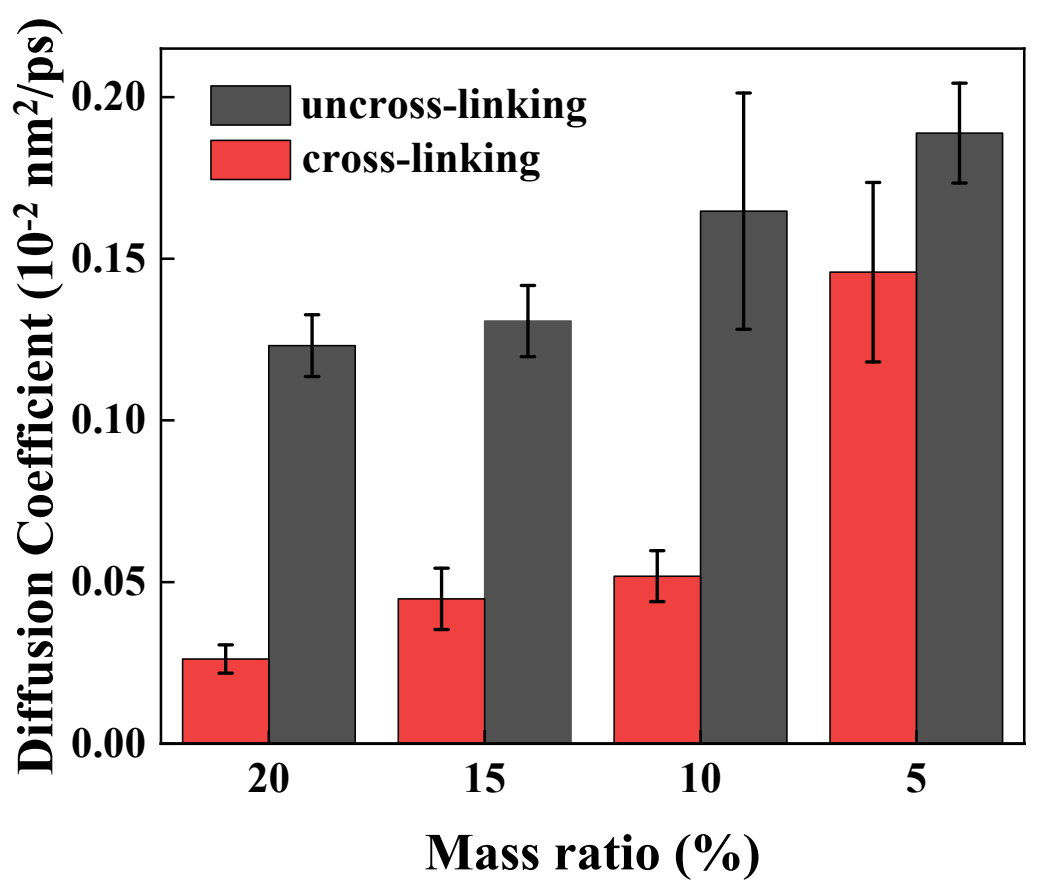




\section{References}

(1) Groot, R. D.; Warren, P. B. Dissipative particle dynamics: Bridging the gap between atomistic and mesoscopic simulation. J. Chem. Phys. 1997, 107, 4423-4435.

(2) Luo, Z.; Jiang, J. Molecular dynamics and dissipative particle dynamics simulations for the miscibility of poly(ethylene oxide)/poly(vinyl chloride) blends. Polymer 2010, $51,291-299$.

(3) Nie, S.; Zhang, X.; Gref, R.; Couvreur, P.; Qian, Y.; Zhang, L. Multilamellar nanoparticles self-assembled from opposite charged blends: Insights from mesoscopic simulation. The Journal of Physical Chemistry C 2015, 119, 20649-20661.

(4) Xie, X. N.; Xu, S. P.; Pi, P. H.; Cheng, J.; Wen, X. F.; Liu, X.; Wang, S. N. Dissipative particle dynamic simulation on the assembly and release of sirna/polymer/gold nanoparticles based polyplex. AIChE J. 2018, 64, 810-821.

(5) Shin, E.; Joo, S. H.; Yeom, M. S.; Kwak, S. K. Theoretical study on the stability of insulin within poly-isobutyl cyanoacrylate (PIBCA) nanocapsule. Mol. Simulat. 2019, 45, 896-903.

(6) Feng, Y. H.; Liu, J. L.; Zhu, D. D.; Hao, Y. Y.; Guo, X. D. Multiscale simulations of drug distributions in polymer dissolvable microneedles. Colloids Surf., B 2020, 189, 110844.

(7) Xu, J. C.; Sun, S. Q.; Wang, Z. K.; Peng, S. Y.; Hu, S. Q.; Zhang, L. J. pH-Induced evolution of surface patterns in micelles assembled from dirhamnolipids: dissipative particle dynamics simulation. Phys. Chem. Chem. Phys. 2018, 20, 9460-9470.

(8) Guan, D.; Feng, S.; Zhang, L. Z.; Shi, Q.; Zhao, S. Q.; Xu, C. M. Mesoscale 
Simulation for Heavy Petroleum System Using Structural Unit and Dissipative Particle Dynamics (SU-DPD) Frameworks. Energ. Fuel. 2019, 33, 1049-1060.

(9) Wang, Y.; Zhu, D. D.; Zhou, J.; Wang, Q. L.; Zhang, C. Y.; Liu, Y. J.; Wu, Z. M.; Guo, X. D. Mesoscopic simulation studies on the formation mechanism of drug loaded polymeric micelles. Colloids Surf., B 2015, 136, 536-544.

(10) De Boulle, K.; Glogau, R.; Kono, T.; Nathan, M.; Tezel, A.; Roca-Martinez, J.-X.; Paliwal, S.; Stroumpoulis, D. A review of the metabolism of 1,4-butanediol diglycidyl ether-crosslinked hyaluronic acid dermal fillers. Dermatol. Surg. 2013, 39, 1758-1766. (11) Xu, Z.; Gao, L.; Chen, P.; Yan, L.-T. Diffusive transport of nanoscale objects through cell membranes: a computational perspective. Soft Matter 2020, 16, 3869-3881. (12) Huang, L. Y.; Yu, Y. S.; Lu, X.; Ding, H. M.; Ma, Y. Q. Designing a nanoparticlecontaining polymeric substrate for detecting cancer cells by computer simulations. Nanoscale 2019, 11, 2170-2178. 